\title{
On the optimal pattern for displacement field measurement: random speckle and DIC, or checkerboard and LSA?
}

\author{
M. Grédiac* \\ B. Blaysat ${ }^{\dagger}$ \\ F. Sur S $^{\ddagger}$
}

February 12, 2020

This is the author-manuscript version of

M. Grédiac, B. Blaysat, F. Sur. On the Optimal Pattern for Displacement Field Measurement: Random Speckle and DIC, or Checkerboard and LSA? Experimental Mechanics, SEM \& Springer, 2020.

DOI: $10.1007 / \mathrm{s} 11340-019-00579-z$

\begin{abstract}
This paper deals with the optimal pattern that can be used to retrieve displacement fields by minimizing the optical residual calculated over small regions of contrasted images. This minimization is generally performed in the spatial domain by processing speckle patterns with DIC. Another option is also considered here. It consists in switching this minimization to the Fourier domain. The benefit is that periodic patterns can be processed, which is generally not possible with DIC. It turns out that the optimal pattern in terms of sensor noise propagation is theoretically the checkerboard if it is correctly sampled, and this pattern is periodic. The reason why checkerboard is optimal is that the image gradient is maximum in this case. In addition, the minimization of the image residual in this case has a quasi-direct solution, which considerably speeds up the calculations. We first recall the basics of the different techniques used in the paper, namely classic subset-based DIC, and a spectral method called Localized Spectrum Analysis (LSA). A recent deconvolution procedure introduced to enhance
\end{abstract}

\footnotetext{
*Université Clermont Auvergne, CNRS, SIGMA Clermont, Institut Pascal, F-63000 Clermont-Ferrand, France michel.grediac@uca.fr

${ }^{\dagger}$ Université Clermont Auvergne, CNRS, SIGMA Clermont, Institut Pascal, F-63000 Clermont-Ferrand, France

${ }^{\ddagger}$ LORIA, UMR CNRS 7503 Université de Lorraine, CNRS, INRIA, Campus Scientifique, BP 239, 54506 Vandoeuvre-lès-Nancy Cedex, France
} 
the metrological performance of DIC and LSA is also briefly recalled and used in this study. Synthetic images are considered to assess in different cases the displacement resolution, as well as other sources of spurious spatial fluctuations observed in the displacement fields such as the pattern-induced bias with DIC. The main conclusion is that using checkerboards instead of random speckles leads to measurements featuring a better compromise between spatial resolution and measurement resolution.

Keywords: checkerboard, deconvolution, digital image correlation, grid method, heteroscedastic noise, localized spectrum analysis, metrology, pattern-induced bias, pattern optimization, speckle, windowed Fourier transform

\section{Introduction}

Digital Image Correlation (DIC), whose basic version only requires minimal surface preparation, is a versatile technique which is now routinely employed in experimental mechanics. There is an active community involved in the improvement of the metrological performance of this measuring technique. A prerequisite to reach this goal is that all the parameters influencing the quality of the final results are clearly identified, and their effect quantified. Most of the papers tackling this topical issue deal with the influence, on the displacement and strain fields, of the various options which can be employed when processing images of the deformed surface. The influence of various settings is also studied in these papers. Studying the nature of the matching function used to represent the displacement within the subsets $[1,2]$, examining the influence of the corresponding settings such as the size of the subsets [3], quantifying the impact of the interpolant used when processing the images of the deformed and/or undeformed specimen to get them in the same coordinate system $[4,5]$, or analyzing how noise in images affects the final result $[6,7]$, are typical examples. On the other hand, studying the influence of the pattern on the quality of the results remains a relatively understudied topic, but recent papers on this subject show that this situation progressively changes. Indeed, the very nature of the pattern really constitutes an influencing parameter $[8,9,10,11]$, and the need for standards on full-field measurement techniques requires that reproducible patterns (or at least patterns featuring similar properties) can be obtained and deposited on specimens, which raises the question of the definition of optimized patterns.

A recent overview on the procedures, which are available to define the best pattern for DIC, has led to combine various existing quality metrics to propose, realize and test different solutions [12]. In this reference, the main point raised by the authors is that preexisting metrics on speckle quality assessment may lead to opposite conclusions when estimating the quality of a given pattern. This justifies combining various metrics to obtain solutions constituting a tradeoff between contradictory constraints. For instance noise propagation to the final displacement maps is minimized if, on average, the norm of 
the gradient of the gray level distributions in the images is maximized. The reason is that predictive formulas for this noise show that it is inversely proportional to the norm of this gradient, [13] for instance. The sum of the square of the subset intensity gradient (SSSIG) over the subset is generally used to globally assess this norm [10, 12, 14]. In this regard, a checkerboard constitutes the best pattern if it is correctly sampled in the images [12]. However the periodicity of this pattern makes it difficult, if not impossible in practice, to find the absolute minimum of the optical residual to deduce the actual displacement by DIC if the true displacement is greater than the period of the periodic pattern. The consequence is that checkerboards are not used in DIC applications, other patterns combining various criteria being considered as optimal $[8,9,10,11,12,15,16,17]$. Recently and as illustrated in Figure 1, it has been shown in [18] that thanks to the Parseval's theorem and some mild assumptions, minimizing the optical residual over small regions to find the displacement at the center of these regions, as local DIC does on speckles in the spatial domain, could be switched advantageously to the Fourier domain in case of a periodic pattern. This led to propose subsequently in [19] a Fourier-based method named Localized Spectrum Analysis (LSA) to process images of checkerboard patterns to retrieve displacement fields. In this reference, it is experimentally evidenced that the noise level in displacement and strain maps obtained with a checkerboard pattern is actually significantly lower than the noise level observed in similar maps obtained from classic grid patterns.

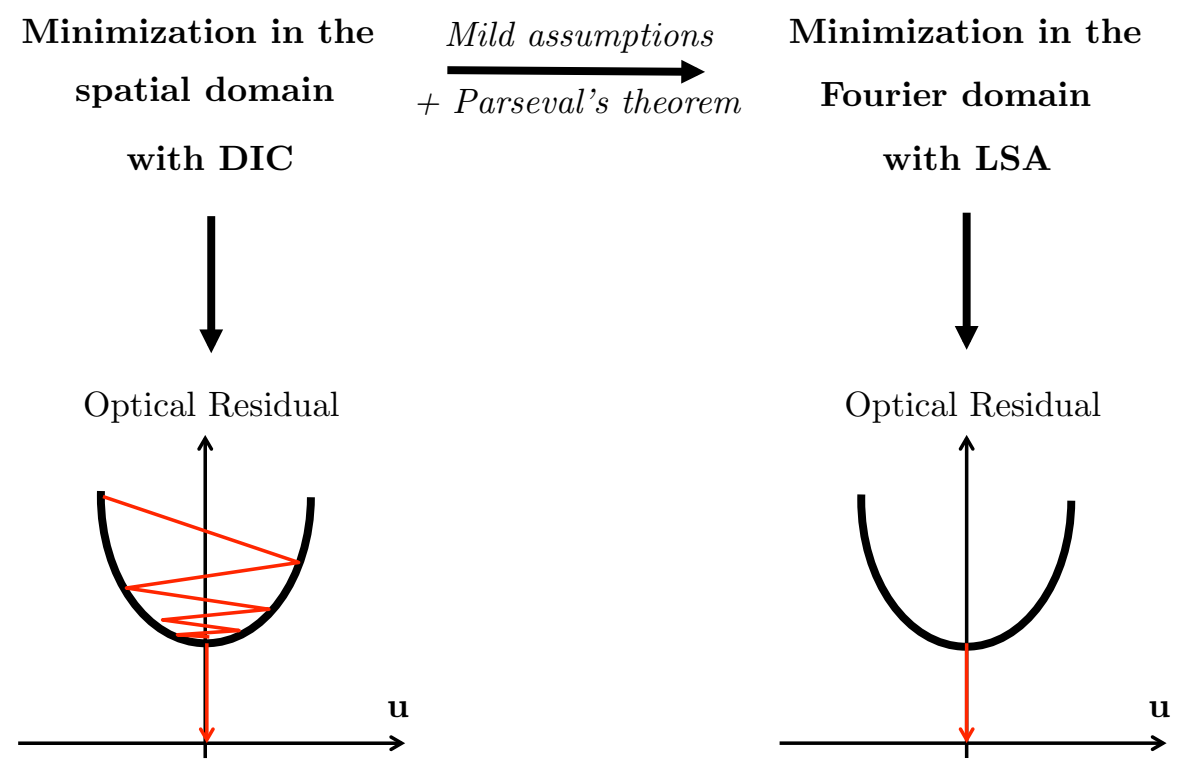

Figure 1: Schematic view of the minimisation of the optical residual in the spatial domain with DIC, and in the Fourier domain with LSA, after the demonstration given in [18]. 
In this context, the objective of the present paper is to compare the quality of the in-plane displacement fields returned by DIC applied on speckles on the one hand, with that observed in displacement fields obtained by using checkerboards and LSA on the other hand. The underlying idea is to check whether checkerboard is really the best pattern to determine displacement and strain fields by minimizing the optical residual, this minimization being performed iteratively with DIC in the spatial domain, and quasi-directly with LSA in the Fourier domain.

The paper is organized as follows. The three metrological parameters considered here to compare DIC and LSA are recalled in Section 2. The tools and the methodology used to compare these two techniques are presented in Section 3. We discuss in Section 4 the results obtained in terms of noise level in the displacement maps, and which is due to sensor noise propagation. Residual bias such as the pattern-induced bias observed in addition to the so-called matching bias in DIC is considered in Section 5. The computing times for DIC and LSA are briefly compared in Section 6. Some experimental results are finally discussed in Section 7.

\section{Definitions and techniques used in the present study}

The definition of the main metrological parameters discussed here is first recalled in this section.

Three metrological parameters are used in this paper. They are defined as follows:

Measurement resolution: in Ref. [20], the measurement resolution is denoted by $\sigma_{u}$ and defined by the smallest change in a quantity being measured that causes a perceptible change in the corresponding indication. More precisely, it is proposed in [21] to define it as the change in quantity being measured that causes a change in the corresponding indication greater than one standard deviation of the measurement noise, which enables us to quantify the measurement resolution. This definition is quite arbitrary, any other (reasonable) multiple of the standard deviation being also potentially acceptable, but the idea is that the resolution quantifies the smallest change not likely to be caused by measurement noise [21].

Spatial resolution: the spatial resolution denoted by $d$ is defined here by the lowest period of a sinusoidal deformation that the technique is able to reproduce before losing a certain percentage of amplitude, this quantity being chosen a priori [22]. The advantage of this definition is that it is not based on an arbitrary value of the subset size in DIC, or of the window used while processing a periodic pattern with LSA (see Section 3.2 below). This makes it possible to compare the spatial resolution between these two techniques, whose principle is totally different. With LSA, this definition of the spatial resolution holds for the phase, and consequently for the displacement. For both techniques, this definition also holds for the strain components if no smoothing is performed before differentiating the displacements. Otherwise the spatial resolution is all the more impaired as the width of the filter is large. 
Bias: a systematic error generally occurs when a given technique returns actual details in displacement and strain maps. It is due to the fact that the amplitude of such apparent details is generally lower than the amplitude of the actual details. This apparent "damping" is a bias, which can be quantified by considering a sinusoidal reference displacement field, and measuring the relative loss of amplitude exhibited by the displacement field returned by the technique under study, as suggested in Refs. [3, 23, 22, 24] for DIC or in $[25,26]$ for LSA. Of course, this relative loss of amplitude, denoted here by $\lambda$. depends on the frequency $f$ of the sine function. In this context, the spatial resolution $d$ is defined for a given bias $\lambda$, the relation between $d$ and $\lambda$ being that $d$ is the smallest value such that the relative loss of amplitude is equal to $\lambda$. In the remainder of this paper, we call $\lambda$ the bias of the method. This is a slight abuse of language since fixing $\lambda$ does not mean that the damping of any displacement or strain field is actually equal to this value of $\lambda$. Note finally that for DIC, the effect quantified here by $\lambda$ is often referred to as the "matching bias", because it occurs when there is a mismatch between the actual displacement and the matching function used to describe this displacement within subsets

\section{Methods}

We propose here to briefly recall the main features of the procedures and tools used in this paper.

\subsection{Digital image correlation}

The classic local version of DIC has been implemented and used in this study [27]. The subset is a square. Its dimension lies between $9 \times 9$ and $41 \times 41$ pixels. The well-known sum of squared differences SSD criterion is chosen for the minimization of the optical residual in the spatial domain. First- and second-order matching functions are used to represent the displacement within the subsets. The usual modified Gauss-Newton scheme [28] is implemented to solve the optimization problem, with a convergence criterion defined with the value of the residual calculated for each iteration. The step between two consecutive subsets is equal to one pixel. This enables us to employ deconvolution as an enhancement tool for the results obtained by classic DIC, as explained in Section 3.3 below in the case of first-order matching functions, because DIC returns the true displacement convolved by a Savitzky-Golay (SG) filter [1]. This value of one pixel also ensures a fair comparison with LSA because displacements and strains are calculated pixelwise with this latter technique, as justified in the following section. Finally, this small value enables us to rely on the ultimate metrological performances of DIC to perform this comparison since no extra interpolation is required to elaborate the displacement map from values available at the center of the subsets only. 


\subsection{Localized Spectrum Analysis applied to checkerboards}

We present here the fundamentals of LSA with more details than in the reminder on DIC above, LSA being less popular than DIC. LSA consists first in calculating the Windowed Fourier Transform (WFT) of the image of a periodic pattern for a given frequency. This is the nominal frequency of the periodic pattern. For a checkerboard aligned with the $x_{1}$ and $x_{2}$ axes, it is shown that the periodic pattern which must be considered is that defined by the two lines of diamonds that can be seen along the bisectors of the $x_{1}$ and $x_{2}$ directions [19] (see Figure 2).

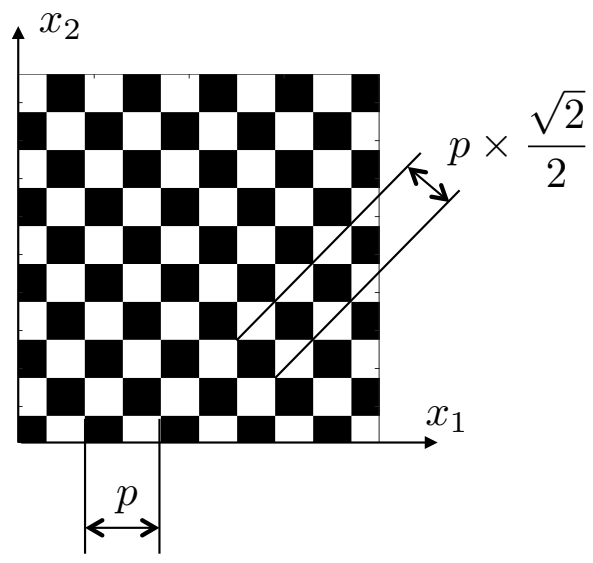

Figure 2: Schematic view of a checkerboard aligned with the $x_{1}-x_{2}$ axes.

The spatial frequency $f$ of the pattern along these directions is $f=\frac{\sqrt{2}}{p}$. In this case, the WFT can be written as follows

$$
\left.\widehat{s_{w}}\left(x_{1}, x_{2}, \theta\right)=\int_{-\infty}^{+\infty} \int_{-\infty}^{+\infty} s\left(\eta_{1}, \eta_{2}\right) w\left(x_{1}-\eta_{1}, x_{2}-\eta_{2}\right) e^{-2 i \pi f\left(\eta_{1} \cos \theta+\eta_{2} \sin \theta\right.}\right) d \eta_{1} d \eta_{2}
$$

where $s$ is the gray level distribution of the image. $w$ is a window centered at the pixel of coordinates $x_{1}, x_{2}$ where $\widehat{s_{w}}\left(x_{1}, x_{2}, \theta\right)$ is calculated. A Gaussian window constitutes the best tradeoff between various constraints [29]. The function defining this Gaussian window is given by the following equation:

$$
w\left(x_{1}, x_{2}\right)=\frac{1}{2 \pi \ell^{2}} e^{\left(-\frac{x_{1}^{2}+x_{2}^{2}}{2 \ell^{2}}\right)}
$$

where $\ell$ is the standard deviation of the Gaussian function. Similarly to $2 M+1$ for the subset size in DIC, $\ell$ can be considered as a handy parameter which governs the apparent 
width of the window used in LSA, this quantity being equal to $6 \ell$ according to the socalled " $3-\sigma$ rule" [30]. The difference is that $2 M+1$ is an integer whereas $\ell$ is a real number. $\widehat{s_{w}}$ is calculated for two perpendicular directions of the periodic pattern. Hence $\theta= \pm 45$ degrees since we deal with a checkerboard [19]. The calculation of the WFT for these two directions provides two distributions of complex numbers. The arguments of these complex numbers are considered, after unwrapping, to be equal to the two phase distributions of the signal along the bisectors of the checkerboard. These two phases are unwrapped and a mere change of basis finally provides the phases along the $x_{1}$ and $x_{2^{-}}$ directions. These two phases are the two components of a vector denoted by $\underline{\Phi}^{\text {ref }}$ for the reference image and $\underline{\Phi}^{\text {cur }}$ for the current (or deformed) one. In the $\left(x_{1}, x_{2}\right)$ coordinate system, the displacement is finally related to these phases $\underline{\Phi}^{\text {ref }}$ and $\underline{\Phi}^{\text {cur }}$ by using the following equation:

$$
\underline{u}\left(x_{1}, x_{2}\right)=-\frac{p}{2 \pi}\left(\underline{\Phi}^{c u r}\left(x_{1}+u_{1}\left(x_{1}, x_{2}\right), x_{2}+u_{2}\left(x_{1}, x_{2}\right)\right)-\underline{\Phi}^{r e f}\left(x_{1}, x_{2}\right)\right)
$$

$\underline{u}$ is involved in both parts of this equation. It is retrieved by using the fixed-point algorithm, which rapidly converges here, one iteration being generally sufficient [31]. The last remark is that the phases obtained with this approach are not exactly equal, regardless of noise, to the true phases. Indeed they are equal, at first approximation, to the latter convolved by a kernel which is the window $w$ used in Equation 1 above. This result, demonstrated in [32], is similar to the one obtained for displacement in DIC, the only difference being the nature of the kernel [19]. It also means that deconvolution can be used as an enhancement tool for the results obtained with LSA, as briefly recalled below.

\subsection{Deconvolution}

Deconvolution is an efficient numerical tool widely used in image processing. It has been recently shown in [33] that a specific deconvolution algorithm can be used to enhance displacement and strain maps obtained with local DIC or LSA. The enhancement is due to the fact that the spatial resolution decreases (thus is improved) while the displacement resolution (thus the noise level) does not increase in the same proportion. This leads the total error (= random error + systematic error) to be lower after deconvolution. This technique is recent for displacement and strain maps, so its influence on the metrological performance will be studied in various cases considered in this study. As recalled above, the quantities obtained with local DIC and LSA are equal to their true counterparts convolved by a kernel known a priori. The deconvolution procedure used here relies on a Taylor expansion of the convolution product between a quantity $q$, which is either the displacement for DIC or the phase for LSA, and the filter corresponding to the technique used, namely the Savitzky-Golay (SG) filter for DIC [1], or a Gaussian function for LSA [32]. The Taylor expansion is carried out up to the second order. This gives the following link between the true value denoted by $\underline{q}^{\text {true }}$, and its counterpart $\underline{q}$ provided by the measurement technique under consideration. 


$$
\underline{q}^{\text {true }}=\underline{q}-\delta \underline{q}
$$

$\delta q$ is a corrective term obtained with the second-order terms of the Taylor expansion. Indeed, it is shown in [33] that the first derivatives do not play any role in the procedure, contrary to the second-order ones. The deconvolution algorithm consists in iteratively updating the corrective term $\delta q$ between $q^{\text {true }}$ and $q$ defined in Equation 4. Since the corrective term is not rigorously equal to $\delta q$ with this iterative approach, we do not retrieve exactly $q^{\text {true }}$ at the end of the procedure, but a quantity close to $q^{\text {true }}$, which is denoted by $\widetilde{q}$. The approximation of the corrective term used during the deconvolution algorithm is denoted by $\delta \widetilde{q}$. This gives

$$
\left\{\begin{array}{rl}
\widetilde{q}^{0} & =\underline{q} \\
\underline{\widetilde{q}}^{\mathrm{it}+1} & =\underline{\widetilde{q}}-\delta \underline{\tilde{q}}^{\mathrm{it}} \\
\text { with } \delta \underline{\widetilde{q}}^{\mathrm{it}} & =\frac{1}{2} \sum_{i=\{u, v\}} \sum_{k, l=1}^{2} \widetilde{q}_{i, k l}^{\mathrm{it}} I_{k l} \underline{e}_{i}
\end{array},\right.
$$

where $\widetilde{q}_{i, k l}^{\text {it }}$ is the second derivative of $\widetilde{q}^{\text {it }}$ with respect to $x_{k}$ and $x_{l}$ at iteration "it". It is estimated in practice by convolution of the map $q$ with a second-order derivative kernel, chosen here as a classic "Mexican hat". $u$ and $v$ are the directions along which $\underline{\underline{q}}$ is determined. For DIC, these directions are directly the $x_{1}$ and $x_{2}$ axes along which the displacement is given. For LSA applied on checkerboard, these directions are the two bisectors of the $x_{1}$ and $x_{2}$ directions defined in Figure 2 [19]. $I_{k l}$ is the second moment of the kernel $w$ involved in each technique (a SG filter for local DIC, and a Gaussian function for LSA), thus

$$
I_{k l}=\iint_{\left(\eta_{1}, \eta_{2}\right) \in \Re^{2}} w\left(\eta_{1}, \eta_{2}\right) \eta_{k} \eta_{l} \mathrm{~d} \eta_{1} \mathrm{~d} \eta_{2}
$$

The flowchart in Figure 3 sums up the deconvolution procedure.

Finally, it is worth remembering from [33] that deconvolution can be applied for a certain frequency range only in order to avoid amplifying the effect of the highest frequencies involved in the signal. For instance, beyond a certain subset size $2 M+1$, the transfer function of the first-order SG filter is negative above a certain cutoff frequency observed to be equal to the inverse of the subset size $\frac{1}{2 M+1}$. It means that the amplitude of a sine displacement returned by local DIC is not only lower than the actual value beyond this threshold frequency, but that it has a wrong sign. In order that the deconvolution algorithm converges, it is shown in [33] that it is relevant to limit this effect by deconvolving $\widetilde{q}$ only for the frequencies lower than this threshold value, which is possible by considering a notch filter in the Fourier domain while calculating the second-order derivatives involved in the corrective term $\delta \underline{q}^{\text {it. }}$. For LSA, no sign change occurs in the transfer function of the 


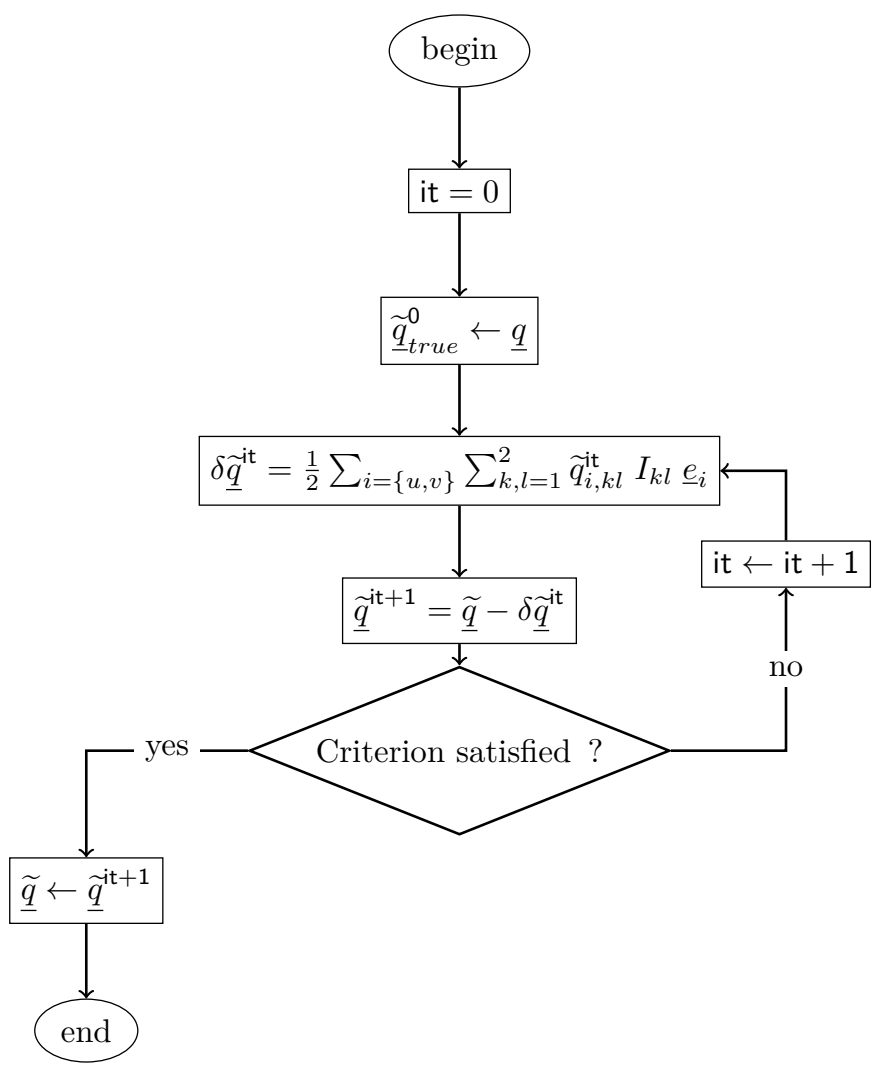

Figure 3: Flowchart of the deconvolution algorithm. $\widetilde{q}$ is the displacement $\underline{u}$ when the minimization of the optical residual is performed on random speckles in the spatial domain (DIC), and the phase $\underline{\Phi}$ when the minimization of the optical residual is performed on checkerboards in the Fourier domain (LSA). In practice the criterion used to stop the iterative calculation of the corrective term is the number of iterations, equal to 10 in the present study. 
Gaussian window used in the windowed Fourier transform since the Fourier transform of a Gaussian function is also a Gaussian function. It has been observed in [33] that for LSA, choosing a threshold value equal to 0.1 pixel $^{-1}$ leads to a reduction of the noise in the maps obtained after deconvolution. In conclusion, only the frequencies lower than a threshold

value equal to $\frac{1}{2 M+1}$ for DIC and $0.1 \mathrm{pixel}^{-1}$ for LSA will be impacted by deconvolution in the results given in this study.

Applying deconvolution as described above leads the systematic error induced by convolution to be canceled out up to a certain cutoff frequency. A consequence is that the spatial resolution of the technique under consideration is significantly improved, whereas the measurement resolution (thus the noise level) does not increase in proportion. This causes the compromise between these two quantities to be dramatically improved. There are however two main limitations for this approach:

1. the subset size for DIC and the size of the Gaussian window for LSA shall be limited. Otherwise, the deconvolution procedure does not converge. For LSA applied on checkerboards, the maximum value of the standard deviation of the Gaussian function $\ell$ that defines the kernel has been observed to be equal to the size of the pitch of the checkerboard, thus $\ell=p$. For DIC, it was not possible to find a clear threshold value since it seems to be dependent of the random speckle pattern itself. $2 M+1=21$ was observed to be the maximum subset size for the particular pattern used in [33].

2. the second moment $I_{k l}$ of $w$ defined in Equation 6 is null when a second-order SG filter is used, thus when second-degree polynomials are employed to describe the displacement within the subsets in DIC. It means that the deconvolution procedure described here will only be applied in the case of first-order matching functions. It is worth noting that DIC results obtained with second-order matching functions could theoretically be deconvolved by considering a Taylor expansion of the convolution product up to the fourth order (the influence of the third order is null), which is however beyond the scope of the present paper. In particular, correctly estimating the fourth derivatives of the signal to be deconvolved is challenging, if not impossible from a practical point of view.

\subsection{Procedure used to compare sensor noise propagation for DIC and LSA}

\subsubsection{Link between bias, spatial resolution and measurement resolution}

The objective here is to propose a route for a fair comparison of the metrological performance of the different techniques considered here by relying on the metrological parameters recalled in Section 2. A problem is however that the size of the subset used in DIC and the size of the window used in LSA are quantities which are arbitrarily and independently chosen by the user within certain limits, and this choice influences the metrological parameters. 
Inspired from recent studies on the metrological performance of various full-field measurement techniques $[18,34]$, the procedure employed here consists in fixing the bias $\lambda$ to the same value for both techniques, and then in examining how the measurement resolution $\sigma_{u}$ evolves as the spatial resolution $d$ progressively changes. This latter quantity increases with the subset size $2 M+1$ (DIC), and with $\ell$ (LSA). It is rigorously demonstrated in [31] and experimentally verified in [18] that the product defined by $\sigma_{u} \times d$ is constant for LSA without deconvolution. The same property is experimentally observed in [18] for local DIC without deconvolution. The idea here is to sweep a range of subset sizes $2 M+1$ for DIC, and a range of standard deviations $\ell$ for LSA, in such a way that the spatial resolution $d$ progressively changes, and to collect the corresponding displacement resolution $\sigma_{u}$ in each case. The set of subset sizes $2 M+1$ investigated here is $\{9,11, \cdots, 39,41\}$, and the set of standard deviations $\ell$ is $\{6 / \sqrt{2}, 5,6, \cdots, 11,12\}$. The first value of the latter set is justified by the fact that the minimum value of $\ell$ that can be used is $p / \sqrt{2}$ [19], where $p$ is the pitch of the checkerboard (see Figure 2), with $p=6$ pixels in the present study, so that each square of the checkerboard is sampled with 3 pixels [35].

\subsubsection{Artificial images}

DIC and LSA were applied on various synthetic images. Synthetic speckles were considered for DIC. They were obtained by using the speckle generator defined in [36]. They also correspond to the images proposed by the DIC Challenge 2.0 [37, 23]. No interpolation is performed while rendering the deformed speckle patterns with this procedure, so interpolation errors that could potentially occur while generating the deformed patterns are avoided. The parameters driving the speckle pattern generator program are chosen to mimic at best a real speckle [36].

A set of reference/deformed checkerboard images was also generated. Such patterns can easily be obtained by using closed-form expressions, but the same procedure as that used for speckles was employed here for the sake of consistency. In particular, both the checkerboard and the speckle images have the same contrast in this study. This is important here since contrast directly governs image gradient, thus noise propagation to the final displacement and strain maps, as can be checked in various paper on sensor noise propagation available in the literature $[38,27,39,6,7]$ for instance. The gray depth in all speckle and checkerboard images is equal to 8 bit. The natural axes of symmetry of the checkerboard were rotated by 10 degrees in order to avoid aliasing in the checkerboard images, as suggested in [40]. It means that the phases are first calculated in this rotated basis by using the procedure described in Section 3.2. The phases are then expressed in the $x_{1}-x_{2}$ basis aligned with the raws of pixels of the camera by using a change of basis. Closeup views of an artificial speckle and an artificial checkerboard are shown in Figure 4.

The synthetic patterns were deformed by using a specific displacement field proposed in [26] and used in recent studies [31, 19], among which the DIC Challenge 2.0 [37, 23]. It is such that the horizontal displacement is null whereas the vertical one is a mere sine 


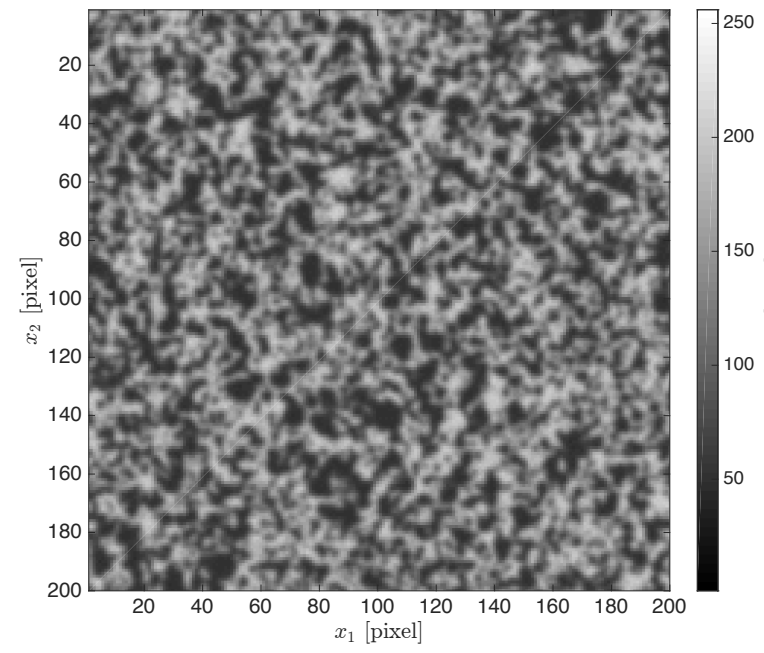

(a) Speckle

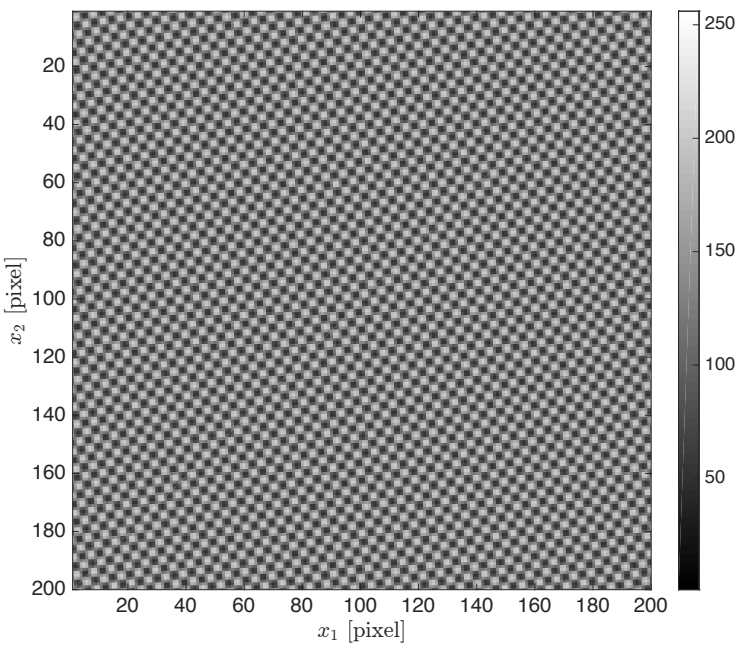

(b) Checkerboard

Figure 4: Closeup views of the synthetic patterns used in this study.

function with a period linearly varying from the left to the right of the image. The midheight also forms a horizontal axis of symmetry along which the displacement is constant and equal to its maximum value, which is here $\left(u_{2}^{r e f}\right)_{\max }=0.5$ pixel. This setting will turn out to be useful below for DIC since the interpolation bias is null for this displacement whatever the nature of the interpolant. Thus

$$
u_{2}^{r e f}\left(x_{1}, x_{2}\right)=0.5 \cos \left(\frac{2 \pi}{p_{\text {wave }}\left(x_{1}\right)}\left(x_{2}-H / 2\right)\right),
$$

where $H$ is the height of the image. The period $p_{\text {wave }}$ is modeled by:

$$
p_{\text {wave }}=p_{\text {wave }}^{\text {mini }}+\frac{p_{\text {wave }}^{\text {maxi }}-p_{\text {wave }}^{\text {mini }}}{L} x_{1},
$$

where $L$ is the length of the image. $p_{\text {wave }}^{\text {mini }}$ and $p_{\text {wave }}^{\text {maxi }}$ are the minimum and maximum values of the period of the sinusoidal displacement, respectively. The different parameters chosen here are $L=2000$ pixels, $H=501$ pixels, $p_{\text {wave }}^{\text {mini }}=10$ pixels and $p_{\text {wave }}^{\text {maxi }}=150$ pixels. Figure 5 shows the distribution of this reference vertical displacement $u_{2}^{r e f}$.

We will see below that plotting the cross-section of the vertical displacement retrieved by the different techniques under study provides meaningful information on their metrological performance.

Finally, noise was added to these synthetic checkerboard and speckle images when image noise propagation was studied. In real cameras, sensor noise is heteroscedastic. It 


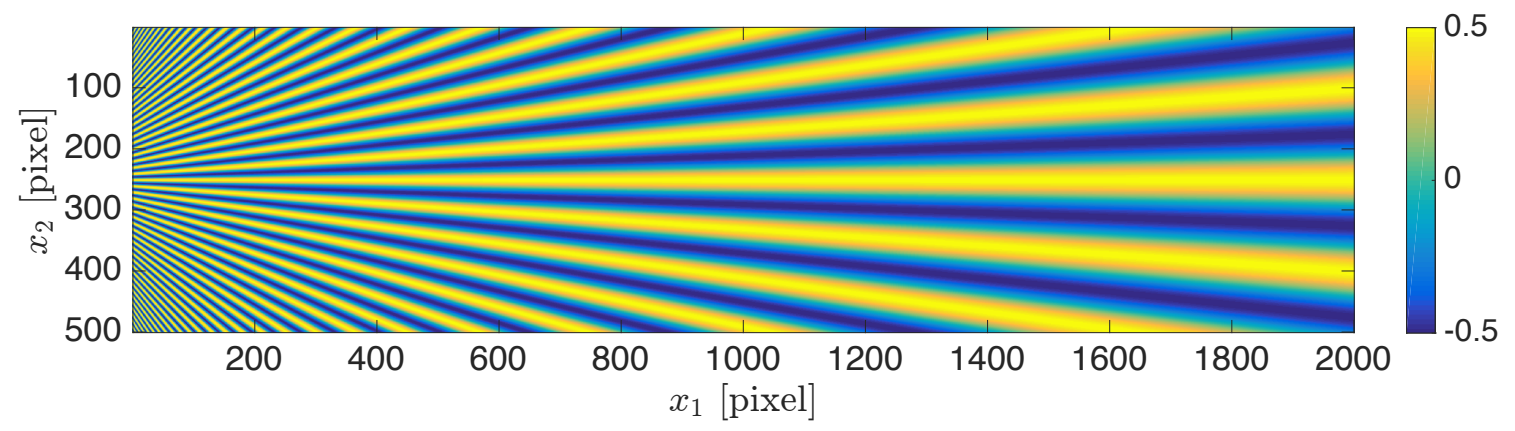

Figure 5: Vertical reference displacement $u_{2}^{r e f}$.

means that its standard deviation $\sigma_{\text {image }}\left(x_{1}, x_{2}\right)$ depends on the gray level $s\left(x_{1}, x_{2}\right)$. This phenomenon is modeled by considering that the variance linearly depends on the gray level [41], so the standard deviation can be written as follows:

$$
\sigma_{\text {image }}\left(x_{1}, x_{2}\right)=\sqrt{a s\left(x_{1}, x_{2}\right)+b},
$$

where $a$ and $b$ are parameters which depend of the camera. In the DIC-challenge, $a=$ $0.0342, b=0.2679$, so these values were also used here to add noise in the checkerboard and speckle images.

\subsubsection{Cases considered in the calculations}

The procedure described above has been applied in different cases which are briefly described below:

- local DIC was applied on the synthetic speckle images deformed with the reference displacement field. Matching functions of degree 1 and 2 were successively considered. The former is frequently implemented in commercial DIC packages, but the latter is expected to more reliably return complex displacement fields. Deconvolution was also applied, but in the former case only. Indeed, results obtained with second-degree matching are not impacted by deconvolution as considered here [33];

- LSA was applied on the synthetic checkerboard images. Deconvolution was also applied to improve the metrological performance of this technique, so results obtained with and without applying deconvolution are presented below;

- DIC is not suited to process periodic patterns, mainly because of convergence issues. They are due to the fact that many local minima appear in the cost function to be minimized. However, if DIC is initialized at a point close to the solution, it is expected to successfully converge. Since we are here in a very particular case for which 
the amplitude of the displacement is not greater than 0.5 pixel, thus lower than half the period of the checkerboard, initiating DIC with a null displacement automatically leads the optimization program to converge toward the correct solution. This would certainly not be possible with real checkerboard images since the displacement obtained during a test is often greater than half the period of the checkerboard, at least at some points. However considering this particular case in the present study enables us to obtain the upper bound of what DIC can offer in terms of sensor noise propagation, checkerboard being the optimal pattern in this regard [12]. This is important because the random speckle pattern considered here is somewhat arbitrary. Because of the random nature of the speckle patterns, any other pattern would potentially lead to results different from the ones found in the particular case here, even by keeping the same setting in the speckle generator. Using here a set of images proposed by the DIC Challenge 2.0 enables us to discuss results obtained with typical speckle images [23]. Refs $[8,9,10,11,12,15,16]$ propose various speckles which can each be considered as optimal with respect to various criteria, but considering all of them is beyond the scope of the present paper. Ref. [12] claims however that any random speckle can not be better than a checkerboard. In the present study, the underlying idea is therefore to estimate to which extent a checkerboard leads to better results than a typical random speckle, and also to observe how DIC performs compared to LSA when applied on exactly the same pattern.

Table 1 sums up the different cases considered here.

\section{Comparison of the techniques and patterns in terms of displacement resolution}

\subsection{Displacement resolution as a function of $2 M+1$ and $\ell$}

The displacement resolution is first assessed in the different cases given in Table 1. It is calculated in each case by considering noisy and noiseless pairs of reference and deformed images, and subtracting the displacement maps obtained in the noisy and noiseless cases, which gives a maps reflecting the noise in the displacement field due to the propagation of the noise affecting the images. "Noise" corresponds here to the random spatial fluctuations of the displacement, which are caused by sensor noise propagation. As expected from Ref. $[38,27,39,6,7]$, it is observed that the frequency of the underlying displacement does not influence this distribution. This is illustrated in a typical example in Figure 6. Some spots can be seen in this noise map because the displacement field is affected by a spatially correlated noise for both LSA and DIC, which means that the typical size of the spots is much greater than one pixel.

The displacement resolution $\sigma_{u}$ is considered to be equal to the standard deviation of this noise estimated over the whole map (excluding the border), as in previous studies, [31] 


\begin{tabular}{|c|c|c|c|c|}
\hline case \# & technique used & $\begin{array}{c}\text { degree of the } \\
\text { matching function }\end{array}$ & pattern & deconvolution \\
\hline 1 & DIC & 1 & SPKL & N \\
\hline 2 & DIC & 1 & SPKL & Y \\
\hline 3 & DIC & 2 & SPKL & N \\
\hline 4 & DIC & 1 & CKB & N \\
\hline 5 & DIC & 1 & CKB & Y \\
\hline 6 & DIC & 2 & CKB & N \\
\hline 7 & LSA & n.a. & CKB & N \\
\hline 8 & LSA & n.a. & CKB & Y \\
\hline
\end{tabular}

Table 1: Cases under study. SPKL: speckle, CKB: checkerboard.

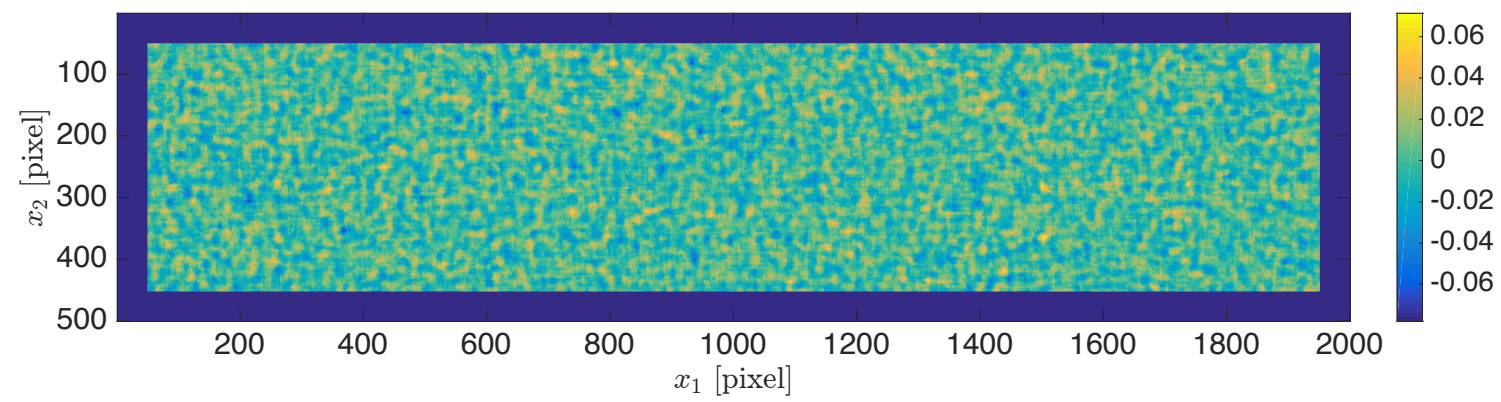

Figure 6: Typical difference between displacement maps obtained with noisy and noiseless pairs of images (in pixel). DIC, subset size $2 M+1=21$ pixels in this example. 
for instance. Figure 7-a shows $\sigma_{u}$ estimated with DIC as a function of the subset size in cases 1, 2 and 3 of Table 1, as well as $\sigma_{u}$ for LSA, as a function of the standard deviation $\ell$ of the Gaussian function in cases 7 and 8. Figure 7-b also compares the results obtained for DIC and LSA, but DIC is applied here on checkerboards, see cases 4, 5 and 6 of Table 1. The results obtained for both techniques are plotted in the same figure. However, at this stage, there is not yet any matching between the subset size $2 M+1$ for DIC on the one hand, and the standard deviation $\ell$ used in LSA on the other hand. This issue will be discussed in the next section. The following comments can be drawn from these curves:

- without deconvolution, $\sigma_{u}$ decreases as the size of the subset and the size of the Gaussian function increase, which is logical, sensor noise being averaged over a larger domain while extracting the displacement from the images;

- when deconvolution is applied, $\sigma_{u}$ is slightly higher than in the preceding case for the lowest values of $2 M+1$ and $\sigma_{u}$. The procedure clearly diverges beyond a certain threshold value equal to the pitch of the checkerboard for LSA, which is consistent with the results found in [33]. Deconvolution is also expected to be unstable for DIC, but the threshold value for the subset size $2 M+1$ beyond which the procedure diverges is not as clear as for LSA. This phenomenon is discussed in [33];

- it can be seen that for a given subset size $2 M+1$, the noise level in the displacement maps is higher for a second-degree matching function than for a first-degree one, which is consistent with the conclusion of [27];

- by comparing the curves found for DIC in Figures 7-a and 7-b concerning DIC, it is also worth noting that the noise level is much higher for DIC applied on speckle than for DIC applied on checkerboard. The ratio between these two quantities, plotted in Figure 8, is quite significant since it lies approximately between 2.3 and 3.3. This ratio is observed to be the lowest for the largest subset sizes. This result is consistent with the conclusion given in [12]. It is worth mentioning that DIC is applied here on checkerboard by initiating the iterative minimization of the optical residual with a displacement equal to zero, which is possible because the amplitude of the reference displacement deforming the pattern is lower than half its period;

- it can be seen in Figure 7-b that LSA applied on checkerboard leads to values of the displacement resolution, which are similar to the ones obtained with DIC applied on checkerboard. By extension, it means that the ratio between the displacement resolutions obtained with DIC applied on speckle, and with LSA applied on checkerboard, has the same order of magnitude as that shown in Figure 8 for DIC only. 


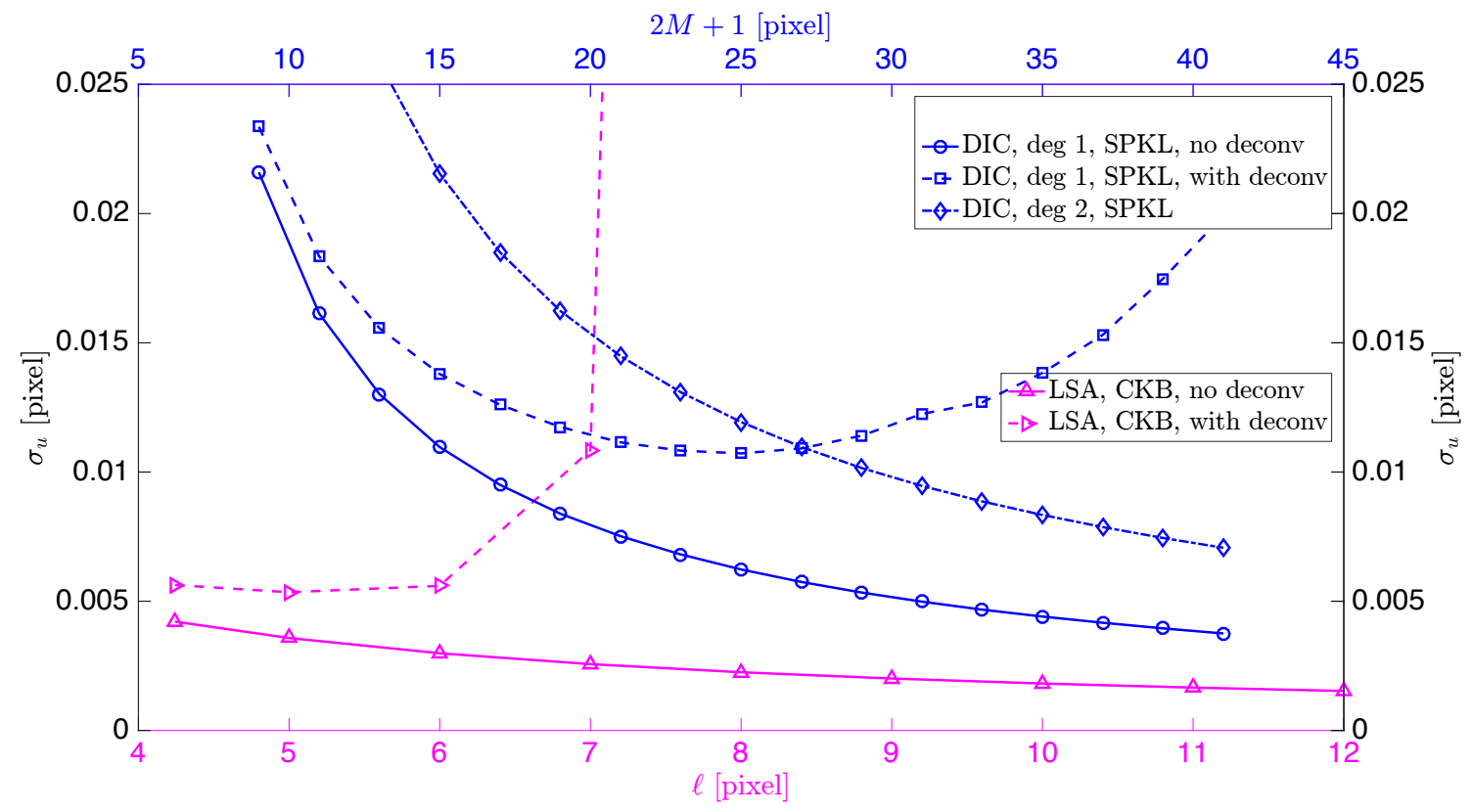

(a) DIC on speckle and LSA on checkerboard

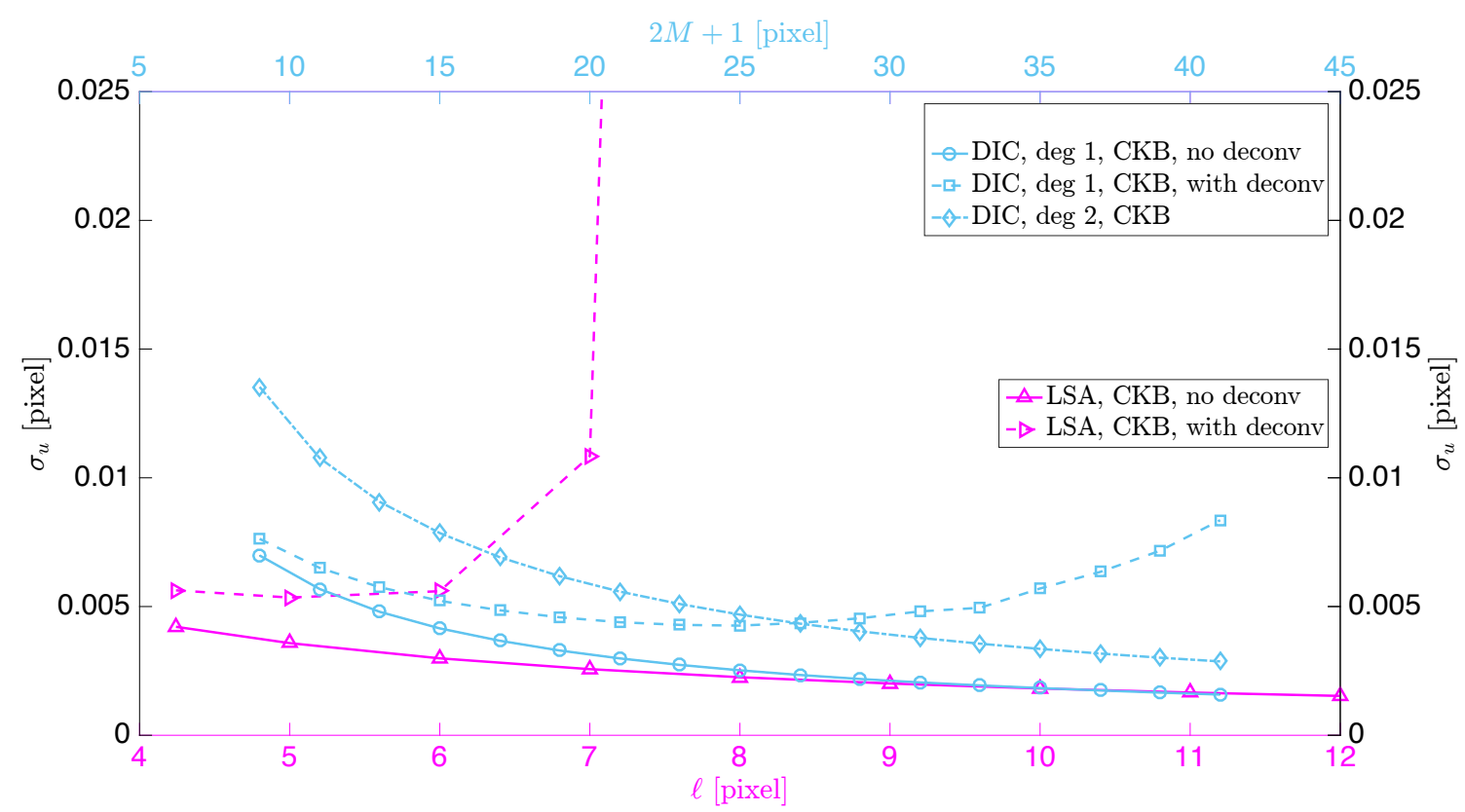

(b) DIC and LSA on checkerboard

Figure 7: Displacement resolution $\sigma_{u}$ as a function of the subset size $2 M+1$ for DIC, or of the apparent size of the Gaussian functionil $6 \times \ell$ for LSA. 


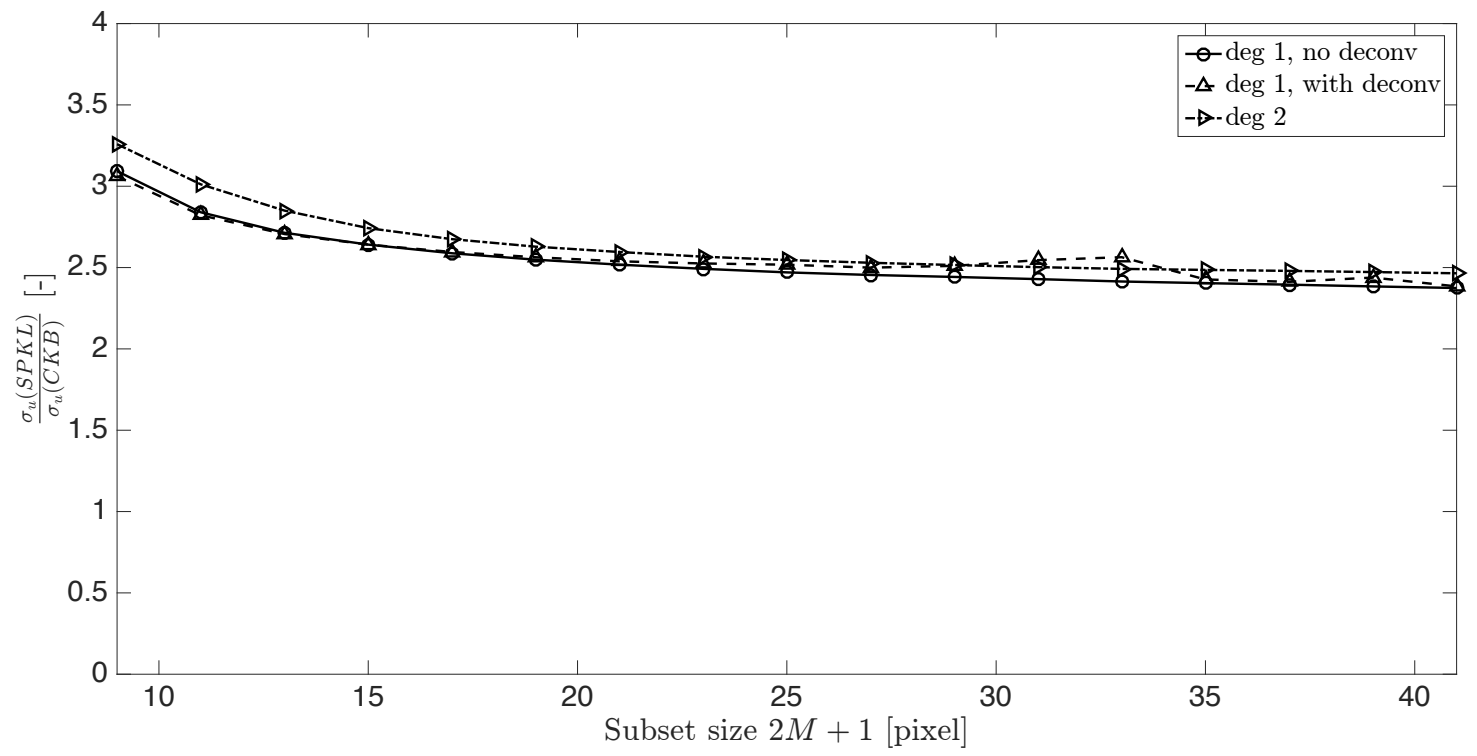

Figure 8: Ratio between displacement resolutions obtained with DIC on speckle and DIC on checkerboard, as a function of the subset size $2 M+1$.

\subsection{Displacement resolution as a function of the spatial resolution $d$}

It is difficult to fairly compare the results given by DIC and LSA from the preceding results because they are given as a function of the subset size $2 M+1$ on the one hand, and on the standard deviation $\ell$ of the Gaussian window $w$ on the other hand, and because these two quantities are not directly comparable. The apparent size of a Gaussian window can be estimated as $6 \ell$ according to the " $3-\sigma$ rule" [30] but this definition is quite arbitrary. In addition, the influence on the final result of the pixels lying in this zone is not uniform. This is the reason why we relied here of an objective quantity, which is the spatial resolution $d$ as defined in [22] and recalled in Section 2. This quantity is estimated in each case from the displacement map returned by the technique used with an image deformed with the reference displacement defined in Figure 5. Maps obtained with noiseless images are used for this determination. The bias $\lambda$ is first fixed to a given (and acceptable) quantity, the period of the sine displacement for which the amplitude returned by the technique is equal to $(1-\lambda)$ times the reference displacement is deduced. $\lambda$ is set here to $10 \%$. In practice, the cross-section of the map along the axis of symmetry is plotted, and the intersection with the horizontal line defined by $u_{2}=\left(u_{2}^{r e f}\right)_{\max }(1-\lambda)=0.5(1-\lambda)$ is determined. The curves being often affected by high-frequency fluctuations due to image noise or to the pattern-induced bias (this phenomenon is discussed in Section 5 below), they are smoothed with an averaging filter of width 200 pixels, in order to find an intersecting 
point less dependent on these fluctuations. This procedure is applied in each case, which gives for each technique and pattern a set of points in the plane defined by the spatial resolution $d$ and the displacement resolution $\sigma_{u}$. The link between the two quantities $\left(\sigma_{u}\right.$ and $d$ ) can be represented by a curve obtained from the calculations carried out with the different values of $2 M+1$ and $\ell$ given above. This curve can be considered as an intrinsic signature of a given technique when applied to a pair of reference and deformed images. In addition, if, as expected, the product $\sigma_{u} \times d$ is really constant [18], considering a logarithmic scale for both quantities, as in [34], should lead to a straight line of slope -1 in the $\sigma_{u}$ - plane. This enables us to easily visually observe whether this property (the fact that $\sigma_{u} \times d$ is constant) is satisfied or not. We start first with the results obtained with DIC applied on the speckle and checkerboard images. The corresponding curves are depicted in Figure 9 (the points corresponding to the cases for which $d \geq 150$ pixels are not represented in this figure)

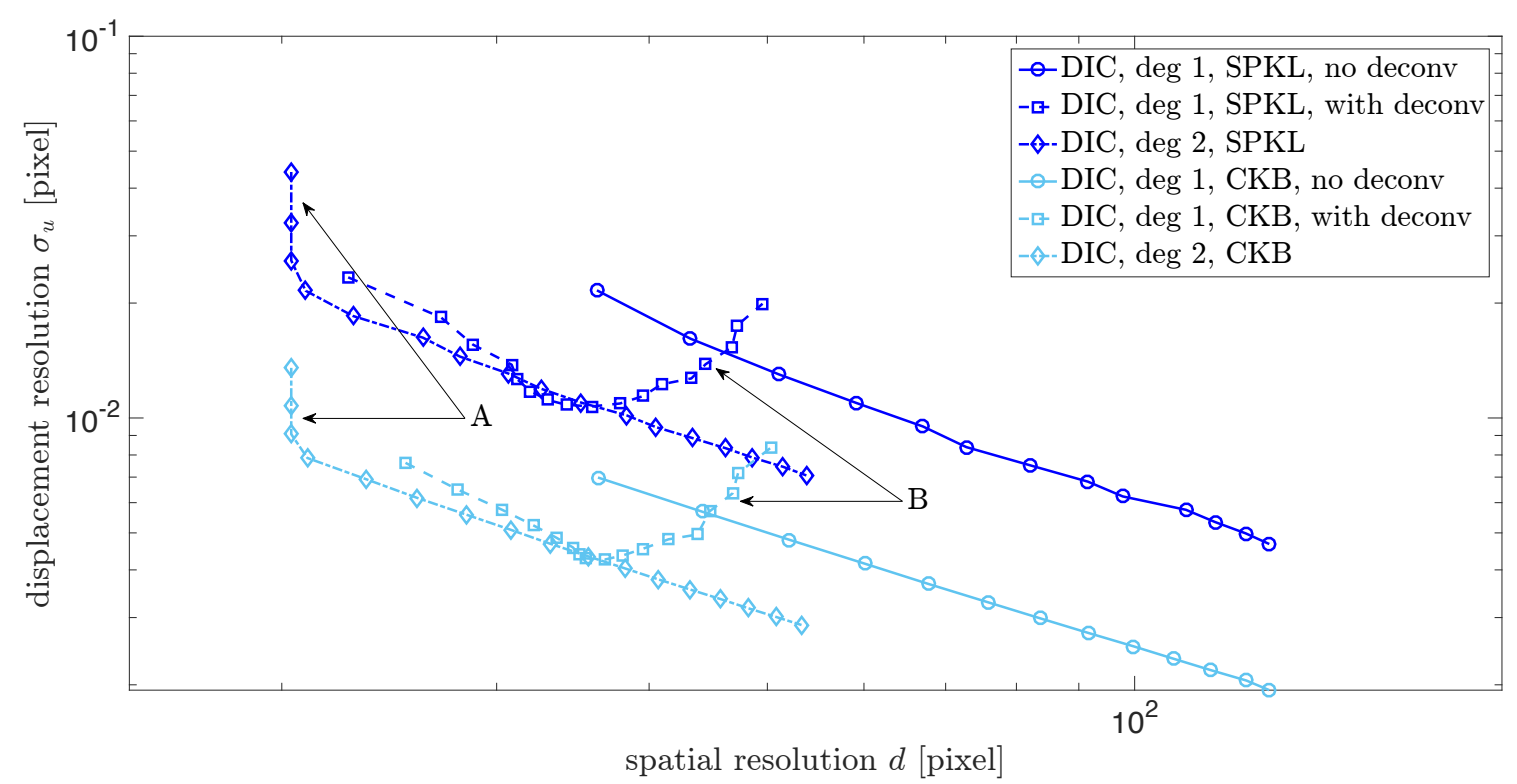

Figure 9: Displacement resolution $\sigma_{u}$ as a function of the spatial resolution $d$ for DIC applied on speckle and on checkerboard.

The first striking point is that nearly the same sets of curves are obtained in both cases, but they are shifted toward the bottom for the checkerboard, which means that the displacement resolution (and thus the noise level) is lower in the second case. In theory, we should find exactly the same spatial resolution for a given subset size whatever the pattern encoding the actual displacement (random speckle or checkerboard). Hence, when assessing $d$ by determining the abscissa of the intersection between the cross-section of the map along 
the axis of symmetry on the one hand, and the horizontal line defined by $u_{2}=0.5(1-\lambda)$ on the other hand, we should find the same result for the checkerboard and for the speckle, which is not rigorously the case. This slight difference is due to the fact that the curves are smoothed with an averaging filter of width 200 pixels while finding numerically the spatial resolution in order to reduce the influence of the apparent high-frequency fluctuations impacting the curves. Figure 10-a shows the curves used in a the particular case "DIC with first-order matching functions", with $\lambda=0.1$ and $2 M+1=21$ pixels. Speckle and checkerboard images are considered in turn. It can be seen that the results obtained for the two types of patterns are nearly the same, apart from the aforementioned fluctuations which affect the curve obtained for the speckle. A closeup view of the same curves near their crossing points with the horizontal line of equation $u_{2}=0.5(1-\lambda)$, is given in Figure 10-b. It can be seen that the intersection points with the smoothed curves are not exactly the same. This leads the points characterizing the two types of patterns not to be perfectly aligned along the vertical direction in Figure 9.

Additional comments can also be drawn from the curves shown in Figure 9:

- when no deconvolution is applied, the points line up very well along different inclined straight lines, except some points located at the very left of the figure which are aligned along a vertical line (see Region A). These points correspond to the lowest values of $2 M+1$ used with second-degree matching functions. This local non-linearity of the response is certainly due to the fact that the size of the speckles is not sufficiently small compared to the size of the subset. It means that beyond a certain value, reducing the size of the subset does not bring any added value in terms of improvement of the spatial resolution. The slopes of the curves considered in their linear portion is reported in Table 2 . They were calculated with the robustfit function of Matlab. It can be seen that the values of this slope are close to -1, which illustrates the fact that displacement resolution and spatial resolution are nearly inversely proportional. Note that a similar result is obtained in [42] in the case of DIC, but with another definition of the spatial resolution.

- considering second-degree matching functions in DIC causes the spatial resolution to be improved, the representative curves being more toward the left of the diagram (see the blue dot-dashed lines) than the curves of the first-degree matching functions;

- the positive effect of deconvolution is clearly illustrated by the fact that the corresponding curves are markedly shifted toward the left (see the dashed lines). It means that the spatial resolution significantly decreases, and thus that it is improved. For the lowest values of the subset size, the points are approximately aligned, which is no longer the case for the highest values (see Region B). This phenomenon illustrates the fact that deconvolution progressively diverges for the highest values of the subset size. 


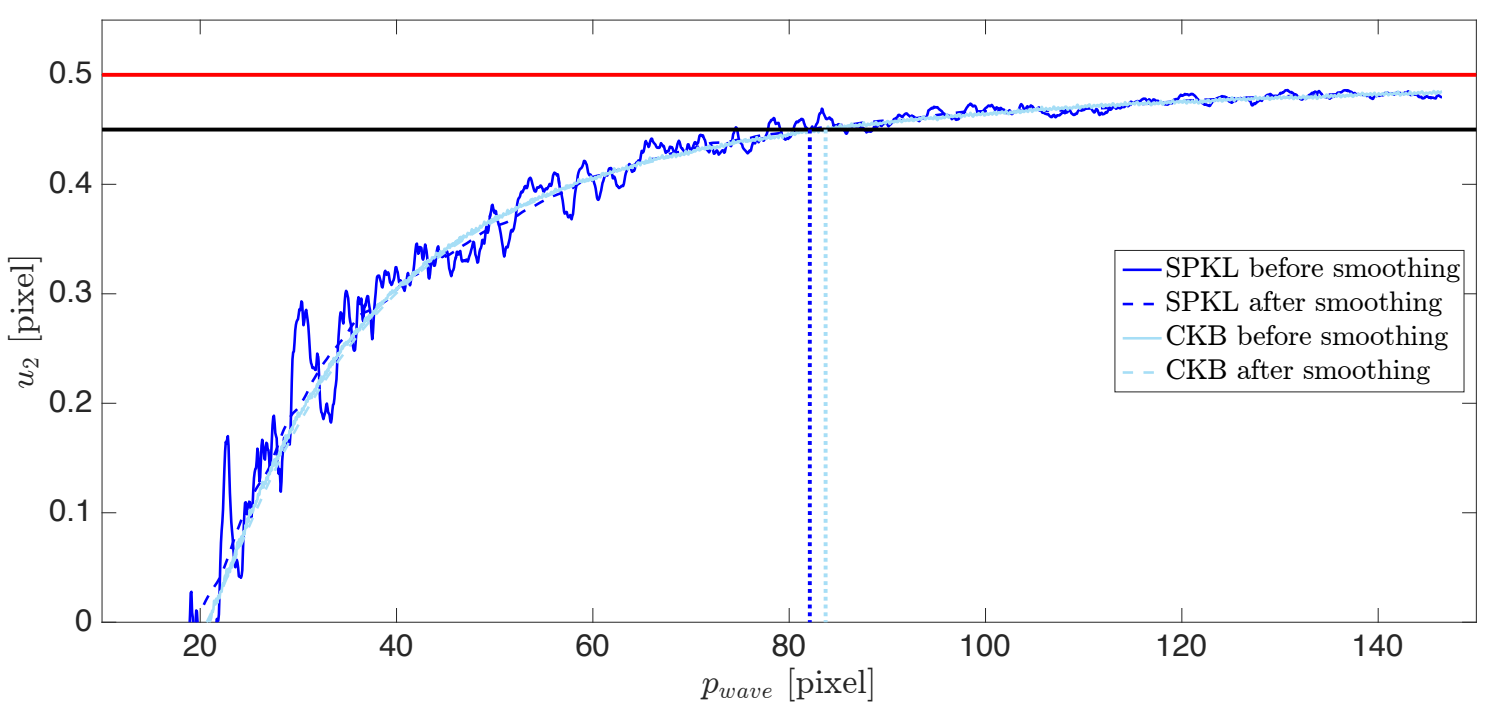

(a)

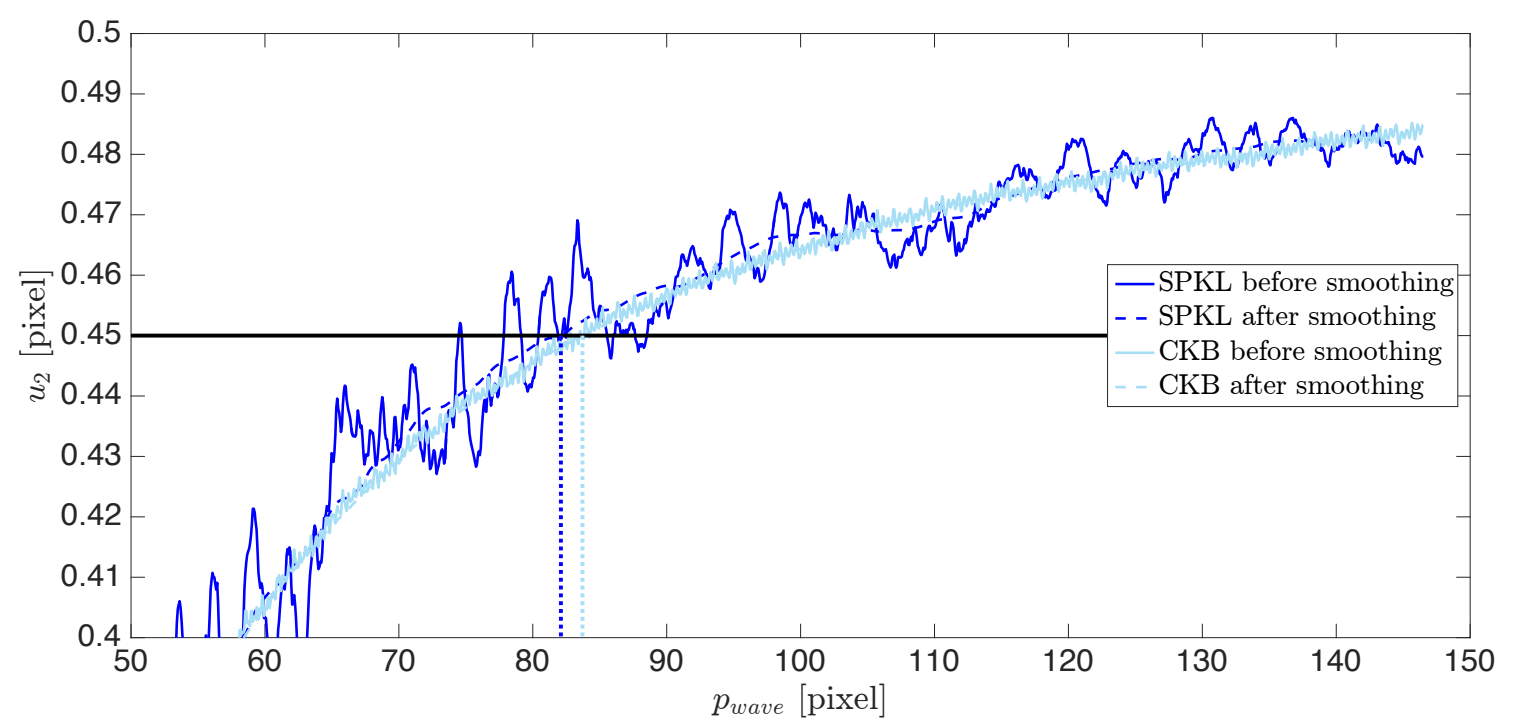

(b) closeup view

Figure 10: Determination of the spatial resolution in a typical case $(\lambda=0.1$ and $2 M+1=$ 21 pixels). DIC on speckle (SPKL) and checkerboard (CKB) with first-order matching functions are considered here. a- cross-section of the displacement field $u_{2}$ along the midline. b- closeup view near the crossing point with the horizontal line $u_{2}=0.5(1-\lambda)=0.45$ pixel. The curves for CKB before and after smoothing are difficult to distinguish, the fluctuations before smoothing being small. 


\begin{tabular}{|c|c|c|c|c|}
\hline case \# & technique used & $\begin{array}{c}\text { degree of the } \\
\text { matching function }\end{array}$ & pattern & slope \\
\hline 1 & DIC & 1 & SPKL & -1.10 \\
\hline 3 & DIC & 2 & SPKL & -1.14 \\
\hline 4 & DIC & 1 & CKB & -0.99 \\
\hline 6 & DIC & 2 & CKB & -1.07 \\
\hline 7 & LSA & n.a. & CKB & -0.98 \\
\hline
\end{tabular}

Table 2: Slopes of the $\sigma_{u}-d$ curves (with logarithmic scale) in their linear portion.

The results obtained with LSA are now added to the previous ones. This gives the curves shown in Figure 11.

- interestingly, DIC applied on checkerboard with first-degree matching functions is equivalent to LSA, the representative curves being superimposed in these two cases (see Region C). However, DIC enables us to reach lower spatial resolutions than LSA, the minimum acceptable value of $\ell$ leading to a higher spatial resolution than the lowest value of the subset size considered here with DIC;

- the preceding remark is no longer valid after deconvolution, the bright blue dashed curve being located less toward the left than the pink dashed line (see Region D);

- in the curves which are discussed here, both the subset size $2 M+1$ for DIC and the standard deviation of the Gaussian function $\ell$ for LSA increase when going toward the right. There is however an exception. This is the case for which deconvolution is applied on LSA. This case corresponds to the pink dashed curve in Figure 11. In this case, the procedure converges only if $\ell \leq p$, namely only if the standard deviation of the Gaussian window is lower than the pitch of the checkerboard. As a consequence, only the points corresponding to the lowest values of $\ell(\ell \leq 7$ pixels) are plotted in this figure. Counterintuitively, the spatial resolution is better for $\ell=p$, which is the highest value of $p$ for which deconvolution converges, than for $\ell=p \frac{\sqrt{2}}{2}$, which is the lowest reasonable value of $\ell$ suggested in [33]. Consequently, the standard deviation $\ell$ of the Gaussian window used with LSA increases when going toward the left on the a pink dashed curve. 


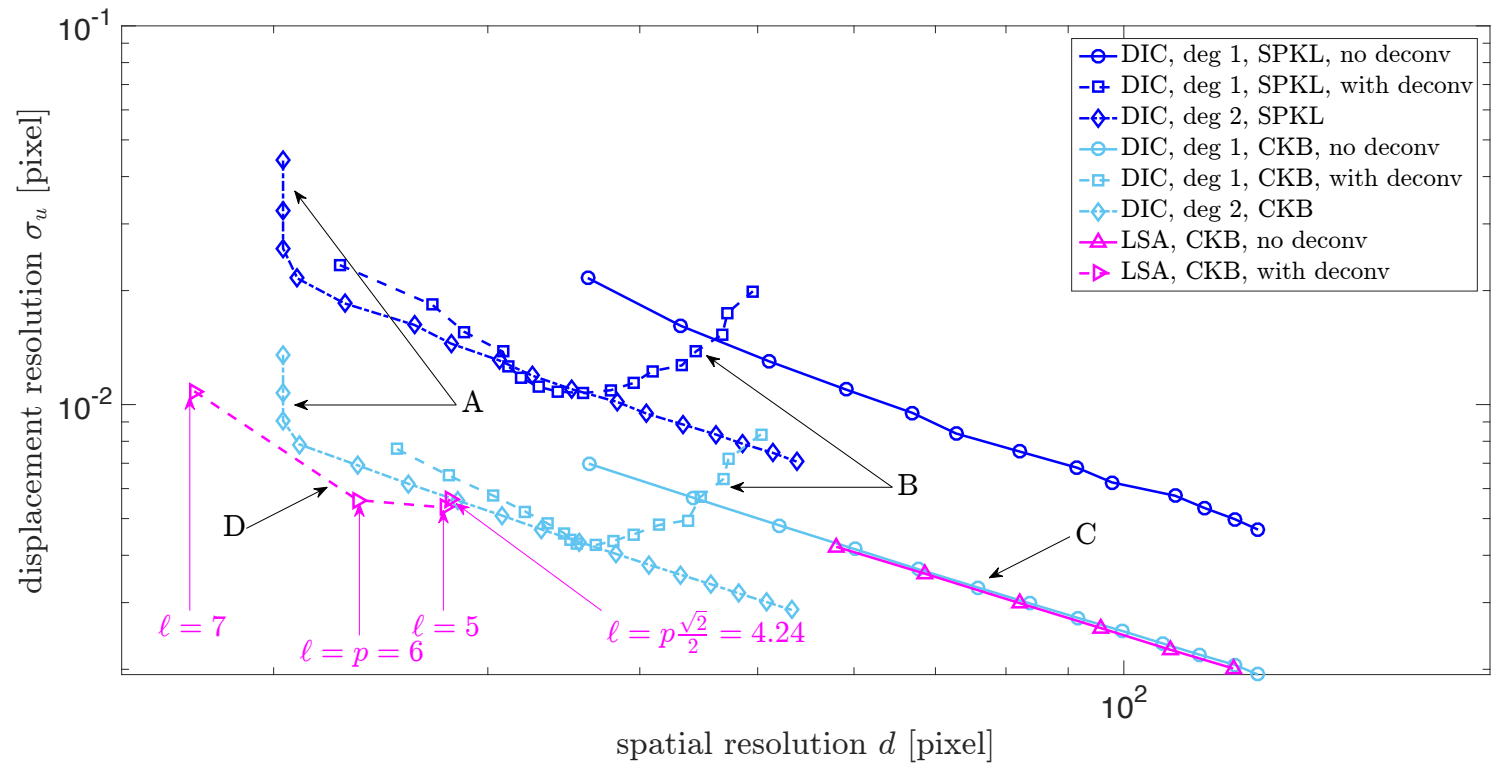

Figure 11: Displacement resolution $\sigma_{u}$ as a function of the spatial resolution $d$ in the different cases studied in Figure 7 . The size of the subset size $2 M+1$ or the Gaussian window $\ell$ increases for each curve when going from the left to the right, apart from the case "LSA, CKB, with deconv" denoted by D, for which the contrary holds true, see the values of $\ell$ (in pixel) reported in the figure. 
In conclusion, it is confirmed that at iso-spatial resolution, the displacement resolution obtained with a checkerboard is significantly lower than that found with a speckle. DIC could be performed here on checkerboard because the displacement remains lower than 0.5 pixels throughout the deformed images, so lower than half the period of the checkerboard, and it is found that DIC and LSA have a similar performance when applied on this pattern.

\section{Pattern-induced bias and other biases remaining after sub- tracting the effect of convolution}

\subsection{Introduction}

As recalled in Sections 3.1 and 3.2, the quantities returned by DIC and LSA, namely the displacement and the phases, are equal to their true counterparts convolved by a kernel, which is clearly identified. This kernel is a Savitzky-Golay filter $[1,43]$ for local DIC, and the window $w$ used in the WFT for LSA [32], here a Gaussian function. This property is however satisfied at the first order only. Regardless of noise, other phenomena also induce spurious fluctuations in the displacement fields. For instance, it is well-known that interpolation induces an error in DIC calculations [4]. Pattern itself also causes some spatial spurious fluctuations called pattern-induced bias to appear in the displacement fields, but this phenomenon has only been recently addressed in the DIC literature [44, 45, 46, 47]. Finally, it is also worth remembering that the true signal is quantized. This also causes an irreversible loss of information, which propagates up to the final displacement maps. The idea here is to investigate these effects by considering the quantity returned by each technique, and by subtracting from them the quantity convolved by the corresponding kernel. An SG filter is considered with DIC and the quantity to be convolved is directly the displacement. A Gaussian window is considered with LSA and the quantity to be convolved in this case is the phase of the periodic pattern, the displacement being then deduced from the convolved phases by using Equation 3. Images deformed by a given displacement field are required to serve as a reference. The reference displacement field defined in Section 3.4.2 is used here. Then we consider the residual displacement field $\delta \underline{u}^{D I C}$ and $\delta \underline{u}^{L S A}$ defined by the following equations

$$
\left\{\begin{array}{l}
\delta \underline{u}^{D I C}=\underline{u}^{D I C}-\underline{u}^{r e f} * w_{S G} \\
\delta \underline{u}^{L S A}=\underline{u}^{L S A}-\underline{u}^{\text {I }}\left(\underline{\Phi}^{r e f} * w_{L S A}\right)
\end{array},\right.
$$

where $\underline{u}^{D I C}$ is the displacement field returned by DIC by using a pair of synthetic noiseless images, and $\underline{u}^{L S A}$ is the same quantity obtained by LSA. Symbol "*" represents the convolution product of two functions. $\underline{u}^{r e f}$ is the reference displacement defined in Equa-

tion 7. $\underline{u}\left(\underline{\Phi}^{r e f} * w_{L S A}\right)$ is the displacement obtained by solving Equation 3, in which the phases are convolved by the same Gaussian window as that used when calculating $\underline{u}^{L S A}$. 
$\underline{u}\left(\underline{\Phi}^{r e f} * w_{L S A}\right)$ is not rigorously equal to $\underline{u}^{r e f} * w_{L S A}$ because of the fixed-point algorithm used to deduce the displacement from the phases with LSA if it is used with more than one iteration. $\delta \underline{u}^{D I C}$ and $\delta \underline{u}^{L S A}$ are expected to represent, as least for the representative displacement field considered in this study, the deviation of DIC and LSA from the theoretical predictions given in [1] for DIC and [32] for LSA. These predictions claim that both these techniques return at the first order the actual quantity (displacement or phase) convolved by a kernel perfectly identified. In other words, $\delta \underline{u}^{D I C}$ and $\delta \underline{u}^{L S A}$ represent a residual bias, which shall be added to the bias in the displacement field reflected by the quantity $\lambda$ discussed in the preceding sections. The objective here is to compare this residual bias observed for both techniques by considering again the reference displacement field. It is clear that we assume here that the images deformed by the reference displacement are reliable enough so that they do not induce, during the image generation step, any significant additional error compared to the ones which are expected to be characterized. In this regard, it is worth remembering that the image rendering procedure does not rely on any interpolation for the speckle images processed by DIC [36]. A variant of the program available in [36] has been specifically written and used here to rely exactly on the same procedure to render the deformed images in both cases (random speckle or periodic checkerboard).

\subsection{Typical maps of $\delta \underline{u}^{D I C}$ and $\delta \underline{u}^{L S A}$ at iso-bias $\lambda$ and iso-spatial resolu- tion $d$}

\subsubsection{Procedure to find $\ell$ equivalent to $2 M+1$}

To start this discussion, we propose first to show some typical examples of $\delta \underline{u}^{D I C}$ and $\delta \underline{u}^{L S A}$ maps. It is preferable to show these examples in a case for which the settings chosen by the user, namely the subset size $2 M+1$ for DIC and the standard deviation $\ell$ of the Gaussian window for LSA, lead to the same spatial resolution $d$ and the same bias $\lambda$. In the case of first-order matching functions, a closed-form expression demonstrated in the Appendix gives the value of $\ell$ used in LSA, which can be considered as equivalent to the value of $2 M+1$ used in DIC. "Equivalent" means here that both techniques lead to the same spatial resolution $d$ and bias $\lambda$. Hence only the value of $\delta \underline{u}^{D I C}$ and $\delta \underline{u}^{L S A}$ is expected to change from one technique to another, the artificial images being noiseless. Since $M$ is necessarily an integer whereas $\ell$ is a real, the latter shall be governed by the former. As discussed in the Appendix, the following closed-form expression gives the link between these quantities for first-degree matching functions:

$$
\ell=\sqrt{\frac{M(M+1)}{3}} .
$$

However, no similar equation is available for higher-degree matching functions, so a numerical procedure will be used to find the value of $\ell$ equivalent to $M$. It consists in performing the following calculations: 
1. $d$ is first determined for DIC for a given value of the subset size $2 M+1$. The approach based on curve fitting discussed in Section 4.2 is used for this purpose;

2. $d$ is also tabulated for LSA for a set of values of $\ell$ spanning the range over which this latter value is expected to be found. $\left[p \frac{\sqrt{2}}{2}, p\right]$ was used in this study. The same procedure as for DIC, which is based on curve fitting, is used to obtain $d$ as a function of $\ell$ for LSA;

3. the value of $\ell$ corresponding $2 M+1$ is finally found in the table by seeking the value of $d$ for LSA, which is the closest to its counterpart obtained during step 1 for DIC.

Compared to the values obtained with Equation 11, approximate values for $\ell$ are found with this procedure, the error made depending on the sampling of the interval defined in step 2. Figure 12 shows that the difference between the value of $\ell$ found by using Equation 11 and the numerical procedure above is negligible. For instance, for $2 M+1=$ 19 pixels, applying the closed-form solution given in Equation 11 leads to $\ell=5.48$ pixels, while $\ell$ is found to be equal to 5.32 pixels with the numerical procedure. The result obtained after deconvolution is also reported in Figure 12 to highlight the benefit of deconvolution in terms of spatial resolution since this quantity is significantly reduced, see [33] for more details.

\subsubsection{Typical maps without deconvolution}

Figures 13-a, -b and -e show typical $\delta u_{2}^{D I C}$ and $\delta u_{2}^{L S A}$ maps obtained in the cases "DIC applied on speckle and checkerboard, first-order matching function", and "LSA applied on checkerboard". Deconvolution is not considered here. Figures 13-c, -d show the maps obtained with DIC and second-order matching functions. The values of the subset size $2 M+1$ (19 pixels) and the standard deviation of the Gaussian window $\ell$ (5.32 pixels) are such that the spatial resolution $d$ is nearly the same for the three cases shown in Figures 13-a, -b and -e. The difference between the value of $d$ in cases a- and b- $(72.79$ and 75.80, respectively) is due to the procedure illustrated in Figure 10. The values of $d$ in a- and e- (72.79 and 72.93, respectively) are slightly different because $\ell$ corresponding to $2 M+1=19$ pixels is found approximately, as explained just above. The particular value of $2 M+1$ considered here, namely 19 pixels, is chosen because this is the greatest subset size corresponding to a value of $\ell$ that leads to a convergence of the deconvolution procedure for LSA. $2 M+1=21$ pixels corresponds to a value of $\ell$ slightly greater than 6 according to Table 3 in the Appendix, but $\ell=p=6$ pixels is the highest value of $\ell$ for which the deconvolution procedure converges [33]. Two vertical dashed lines are superimposed to all maps. The abscissa of the red one corresponds to the subset size for DIC and to $6 \times \ell$ for LSA. Indeed the latter quantity is the apparent size of the Gaussian window. This gives an idea of the size of the support used to get a measurement at a given pixel, and this size can 


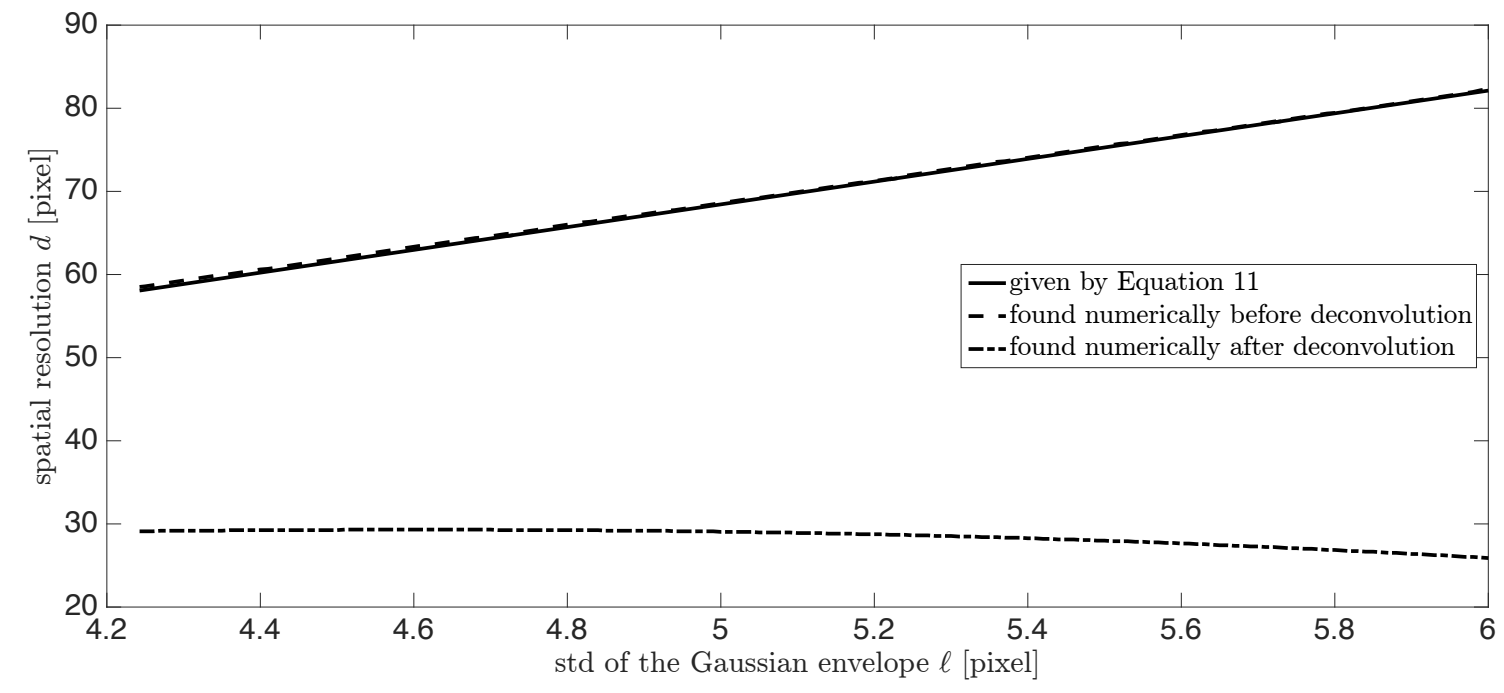

Figure 12: Value of $d$ as a function of $\ell$ in different cases. $\ell$ is bounded by $p \frac{\sqrt{2}}{2}$ and $p$ ( $p=6$ pixels). This is the range for which deconvolution converges for LSA, as explained in $[33]$ 
then easily be compared with the period of the sine wave modeling the displacement since the horizontal axis is graduated in period of the sine displacement wave. The abscissa of the blue dashed line is equal to the spatial resolution $d$ as defined above. It can be checked that, as expected, the latter is nearly the same for the three maps shown in subfigures a, $\mathrm{b}$ and e. Only some slight fluctuations are visible from one case to another. They are due to the procedure used to determine the spatial resolution.

Spurious random fluctuations decorrelated with the displacement encoded in the deformed image are clearly visible on the left-hand side of Figure 13-a and -c. These random fluctuations correspond to the pattern-induced bias described in [44, 46], and modeled in detail in [47]. In [45, 46, 47], it is shown that this bias is much more pronounced when random speckles instead of regular patterns are used, which is confirmed here by comparing Figure 13-a and -c on the one hand, and Figure 13-b and -d on the other hand. It is worth emphasizing that these spurious random fluctuations are still visible on the right-hand side of the vertical blue line whatever the degree of the matching function (see Figure 13-a and -c). It means that actual details in displacement maps having a size of the same order of magnitude as $2 M+1$ are impacted by these spurious fluctuations. It can be observed that the amplitude of $\delta u_{2}^{D I C}$ is more pronounced in the case of second-order matching functions than in the case of first-order ones. This remark holds for the same subset size in both cases, but not for the same spatial resolution, which is lower (thus better) in the second case.

Another phenomenon can be observed in Figure 13-b, -d and -e. Indeed a slight fluctuation correlated with the displacement (and not with the pattern) is visible in these cases. For LSA, (Figure 13-e) and after close inspection (not detailed here), it has been observed that it was due to a slight shift in the phase maps returned by the windowed Fourier transform. Indeed, the phases are theoretically systematically returned with an additive constant equal to $-\frac{\pi}{2}[32]$ but this constant disappears while subtracting the phases obtained for the deformed and reference images. In the present case, this constant is not exactly equal to $-\frac{\pi}{2}$, and the difference between the two changes with an amplitude which is related to the frequency of the sine displacement. A consequence is that a residual appears when subtracting current and reference phase distributions to obtain the displacement. Similar fluctuations related to the displacement are observed with DIC on checkerboard, but no clear explanation was found in this case.

\subsubsection{Impact of deconvolution}

Deconvolution leads these spurious displacements to be enhanced below the cutoff frequency for which this procedure is applied (thus on the right-hand side of the maps), as can be observed in Figure 14, which shows the maps $\delta u_{2}^{D I C}$ and $\delta u_{2}^{L S A}$ after deconvolution of the displacement maps corresponding to the cases shown in Figure 13-a, b and -e. Indeed this spurious displacement is not accounted for in the model used to elaborate the 


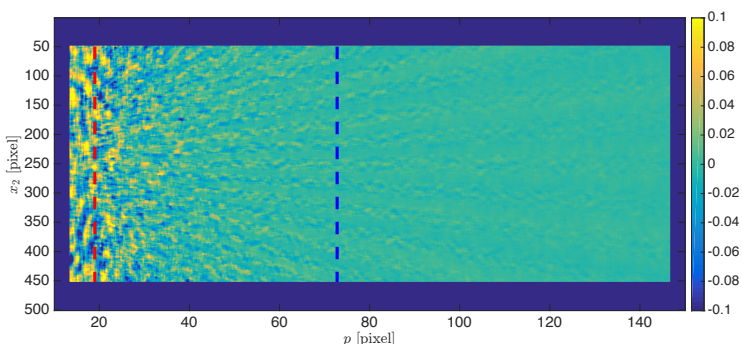

(a) DIC, speckle, first degree, $2 M+1=19$ pixels,

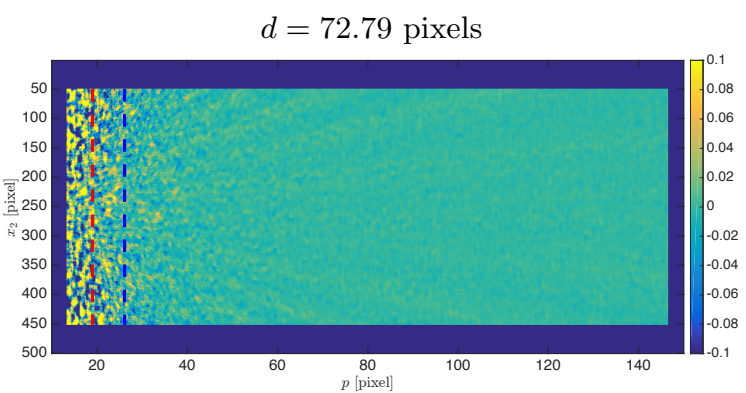

(c) DIC, speckle, second degree, $2 M+1=19$ pixels $d=26.10$ pixels

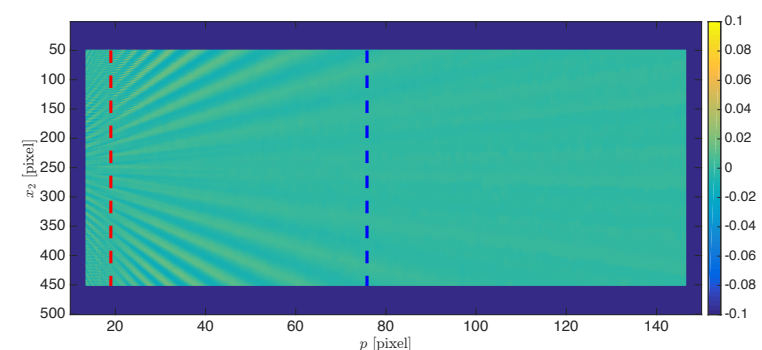

(b) DIC, checkerboard, first degree, $2 M+1=19$ pixels,

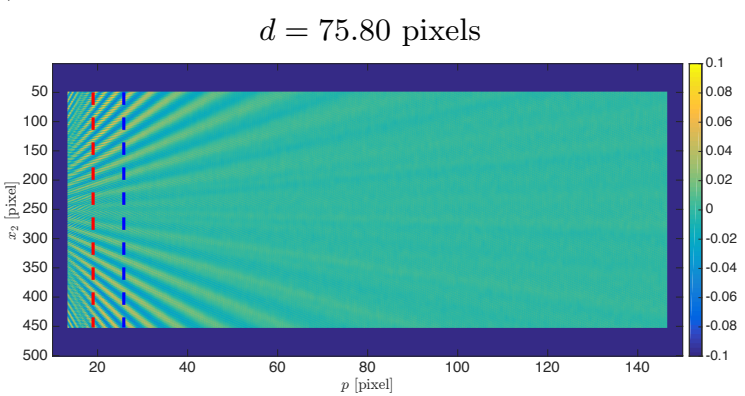

(d) DIC, checkerboard, second degree, $2 M+1=19$ pixels, $d=25.82$ pixels

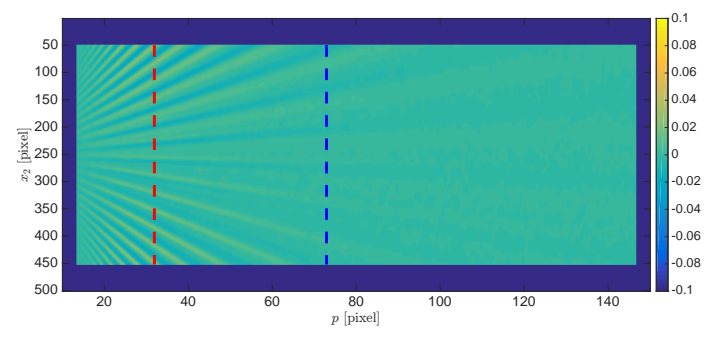

(e) LSA, $\ell=5.32$ pixels, $d=72.93$ pixels

Figure 13: $\delta u_{2}^{D I C}$ and $\delta u_{2}^{L S A}$ in a particular case. First-degree matching functions are used in (a) and (b), and second-degree ones in (c) and (d). The values of the subset size $2 M+1=19$ pixels and standard deviation of the Gaussian window $\ell=5.32$ pixels are such that the same bias $\lambda=10 \%$ and the same spatial resolution $d=72.79$ pixels is obtained in cases (a), (b) and (e). No deconvolution is applied. Abscissa of the vertical red dashed line: $2 M+1$ for DIC and $6 \ell$ for LSA. Abscissa of the vertical blue dashed line: $d$. 
deconvolution procedure [33]. As recalled in Section 3.3, this cutoff frequency is equal to the inverse of the subset size in DIC $\left(\frac{1}{2 M+1}\right)$ and to $\frac{1}{10}$ pixel $^{-1}$ for LSA, as justified in [33].

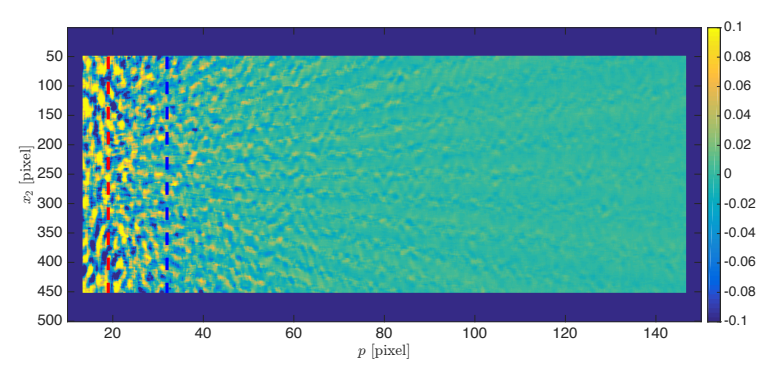

(a) DIC, speckle, first degree with deconvolution, $d=31.98$ pixels

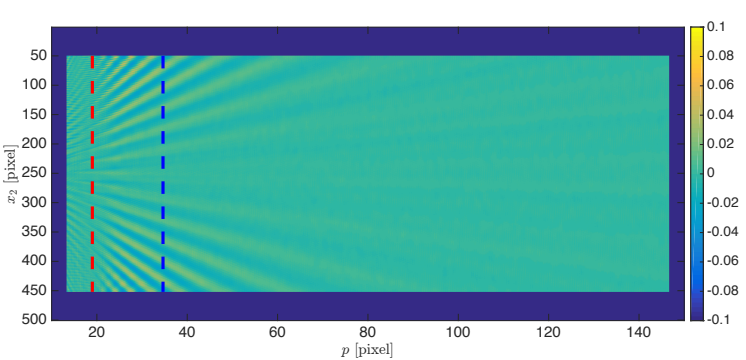

(b) DIC, checkerboard, first degree with deconvolution, $d=34.64$ pixels

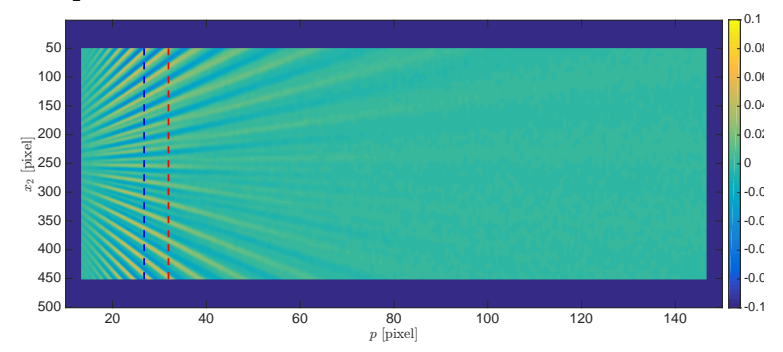

(c) LSA, checkerboard with deconvolution,

$$
d=26.73 \text { pixels }
$$

Figure 14: $\delta u_{2}^{D I C}$ and $\delta u_{2}^{L S A}$ in the same cases as in Figure 13, but after deconvolution. Abscissa of the vertical red dashed line: $2 M+1$ for DIC and $6 \ell$ for LSA. Abscissa of the vertical blue dashed line: $d$.

By comparing the maps shown in Figures 13-a -b -e, and 14-a -b -c, respectively, it can be seen that the amplitude of $\delta u_{2}^{D I C}$ and $\delta u_{2}^{L S A}$ is higher in the second case. The improvement of the spatial resolution is also visible since the vertical dashed blue line is more toward the left after deconvolution. The new location of this line is approximately the same for DIC applied on speckle and on checkerboard images, which is logical since in theory, this location only depends on the kernel involved in deconvolution. However, for LSA after deconvolution, it is even more toward the left, which means that the deconvolution procedure is more efficient for LSA than for DIC in this case.

\subsubsection{Influence of the frequency}

The figures above show that $\delta u_{2}^{D I C}$ and $\delta u_{2}^{L S A}$ depend on the frequency of the displacement. This effect can be quantified by calculating the standard deviation of these quantities column-wise in these maps. Figure 15 shows the results obtained with the same settings as 
above, namely $2 M+1=19$ pixels and $\ell=5.32$ pixels. The standard deviation of $\delta u_{2}^{D I C}$ and $\delta u_{2}^{L S A}$ is plotted as a function of the period $p_{\text {wave }}$ of the sine wave modeling the vertical displacement.

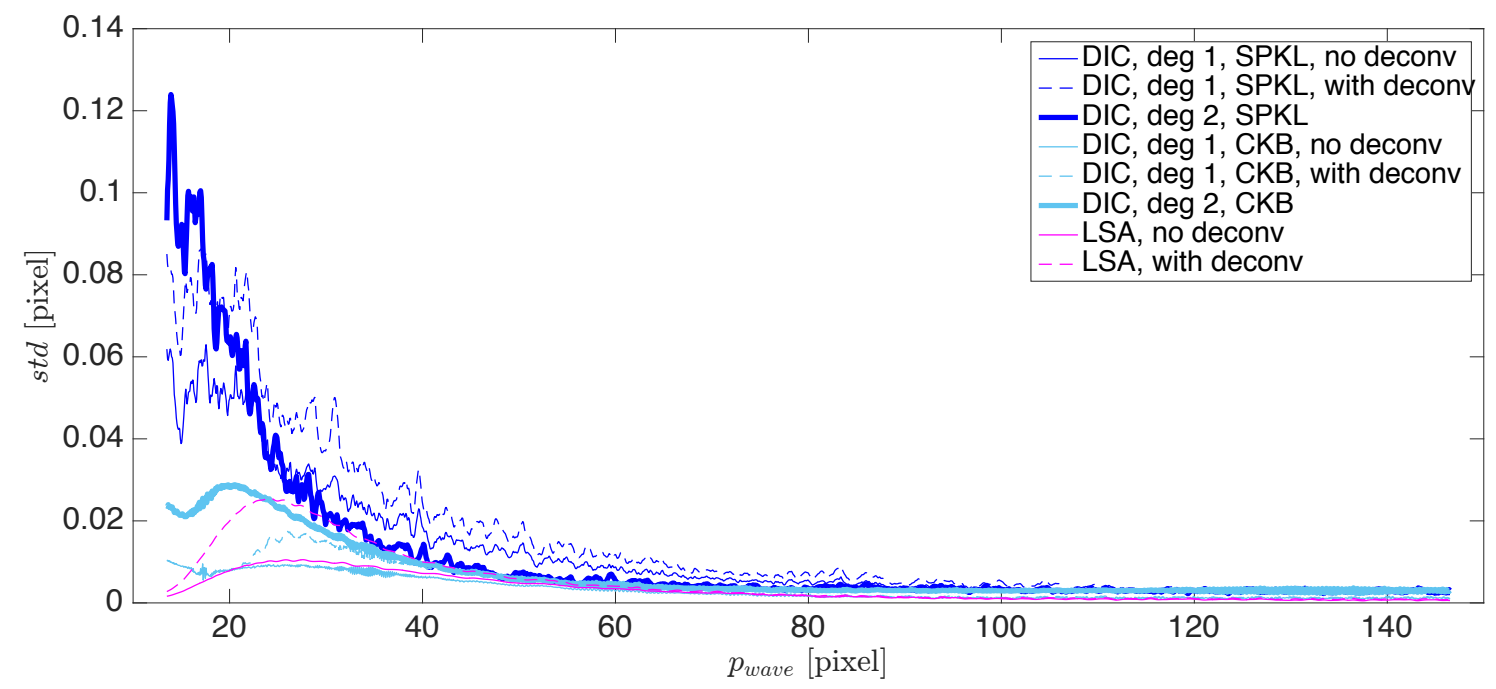

Figure 15: Standard deviation of $\delta u_{2}^{D I C}$ and $\delta u_{2}^{L S A}$ as a function of the period of the wave modeling the displacement. $2 M+1=19$ pixels with DIC and $\ell=5.32$ pixels with LSA.

Some trends are clearly visible in Figure 15:

- $\operatorname{std}\left(\delta u_{2}^{D I C}\right)$ and $\operatorname{std}\left(\delta u_{2}^{L S A}\right)$ globally decrease as $p_{\text {wave }}$ increases, thus as the frequency of the displacement decreases. The only exceptions are at the far left of the curves representing the results for LSA and DIC on checkerboard;

- apart from for the highest frequencies (on the left in the figure), second-degree matching functions lead to a lower standard deviation than first-degree ones in the case of DIC, which is logical, undermatching being one of the main causes of pattern-induced bias, [46, 47];

- as for the displacement resolution $\sigma_{u}$ discussed in the preceding section, $\operatorname{std}\left(\delta u_{2}^{D I C}\right)$ is lower for the checkerboard than for the speckle. The same remark holds for $\operatorname{std}\left(\delta u_{2}^{L S A}\right)$. The difference is significant since the ratio between the two is greater than 2 whatever the frequency of the displacement, as illustrated in Figure 16.

- as expected, deconvolution causes $s t d\left(\delta u_{2}^{D I C}\right)$ and $s t d\left(\delta u_{2}^{L S A}\right)$ to become higher. 


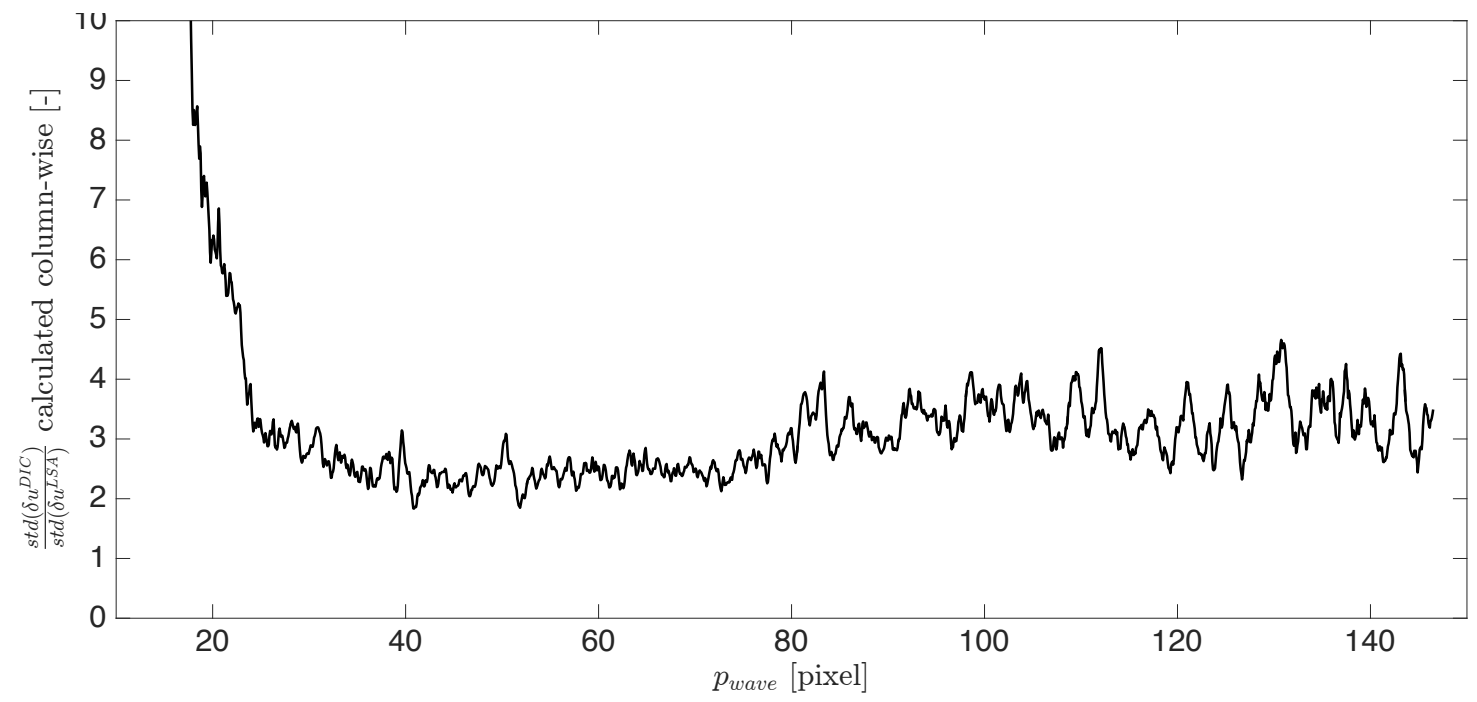

Figure 16: Ratio between the standard deviation of $\delta u_{2}^{D I C}$ and $\delta u_{2}^{L S A}$ calculated columnwise vs. the period of the imposed displacement. The subset size for DIC is equal to $2 M+1=19$ pixels and first-degree matching functions are used. $\ell=5.32$ pixels for LSA, which leads to the same spatial resolution as for DIC. 


\subsection{Equivalent standard deviation of $\delta u_{2}^{D I C}$ and $\delta u_{2}^{L S A}$ as a function of $d$}

In the same spirit as for the displacement resolution discussed in the preceding section, we represent in Figure 17 the equivalent standard deviation calculated over the whole maps of $\delta u_{2}^{D I C}$ and $\delta u_{2}^{L S A}$ in the different cases considered in the simulations in terms of values of $2 M+1$ and $\ell$. These quantities are denoted by $\operatorname{std}_{e}\left(\delta u_{2}^{D I C}\right)$ and $\operatorname{std}_{e}\left(\delta u_{2}^{L S A}\right)$, respectively. The remarks are similar to those drawn for the displacement resolution:

- $\operatorname{std}_{e}\left(\delta u_{2}^{D I C}\right)$ is greater for the speckle pattern than for the checkerboard, which is consistent with the maps represented in Figures 13-14;

- deconvolution shifts the curves toward the left, thus improves the spatial resolution. $s t d_{e}\left(\delta u_{2}^{D I C}\right)$ only increases after deconvolution for DIC on speckle. This quantity remains nearly the same after deconvolution for the other cases;

- the divergence of the deconvolution procedure beyond a certain threshold value of the subset size is illustrated by the set of points which presents an abrupt increase of $\operatorname{std}_{e}\left(\delta u_{2}^{D I C}\right)$ on the blue dashed curve. The same phenomenon is observed with LSA and checkerboard but with a much higher amplitude. The corresponding points being, however, out of the zone considered with the scale of the vertical axis, they are not represented in the figure;

- $\operatorname{std}_{e}\left(\delta u_{2}^{L S A}\right)$ is lower than $s t d_{e}\left(\delta u_{2}^{D I C}\right)$, even when DIC is applied to process checkerboard images.

The values of $\operatorname{std}_{e}\left(\delta u_{2}^{D I C}\right)$ and $s t d_{e}\left(\delta u_{2}^{L S A}\right)$ used in the results shown in Figure 17 were calculated over the whole maps. This clearly disadvantages DIC used on speckles because the highest fluctuations are observed for the highest frequencies, whose weight in real displacement and strain maps is generally not as pronounced as for the synthetic reference displacement considered in this study. In particular, it is generally clear for DIC users that the subset size is such that the actual information characterized by fluctuations with a spatial period lower than the subset size cannot be reliably represented in the maps returned by DIC. The same calculations as those performed above to obtain the results shown in Figure 17 were therefore carried out, but this time by considering only the region of the maps for which the period of the sinusoidal displacement is lower than the spatial resolution of the technique, thus on the right of the blue vertical dashed line plotted in the maps shown in Figures 13-14. Figure 18 shows the results obtained in this case. Compared to the results shown in Figure 17, the curves are now tightened but they exhibit nearly the same shape as above. The results order is also the same as above, which means that minimizing the optical residual in the Fourier domain with checkerboard images globally leads to lower values of $s t d_{e}\left(\delta u_{2}^{D I C}\right)$ and $s t d_{e}\left(\delta u_{2}^{L S A}\right)$ than minimizing the optical residual in the spatial domain on random speckle images. 


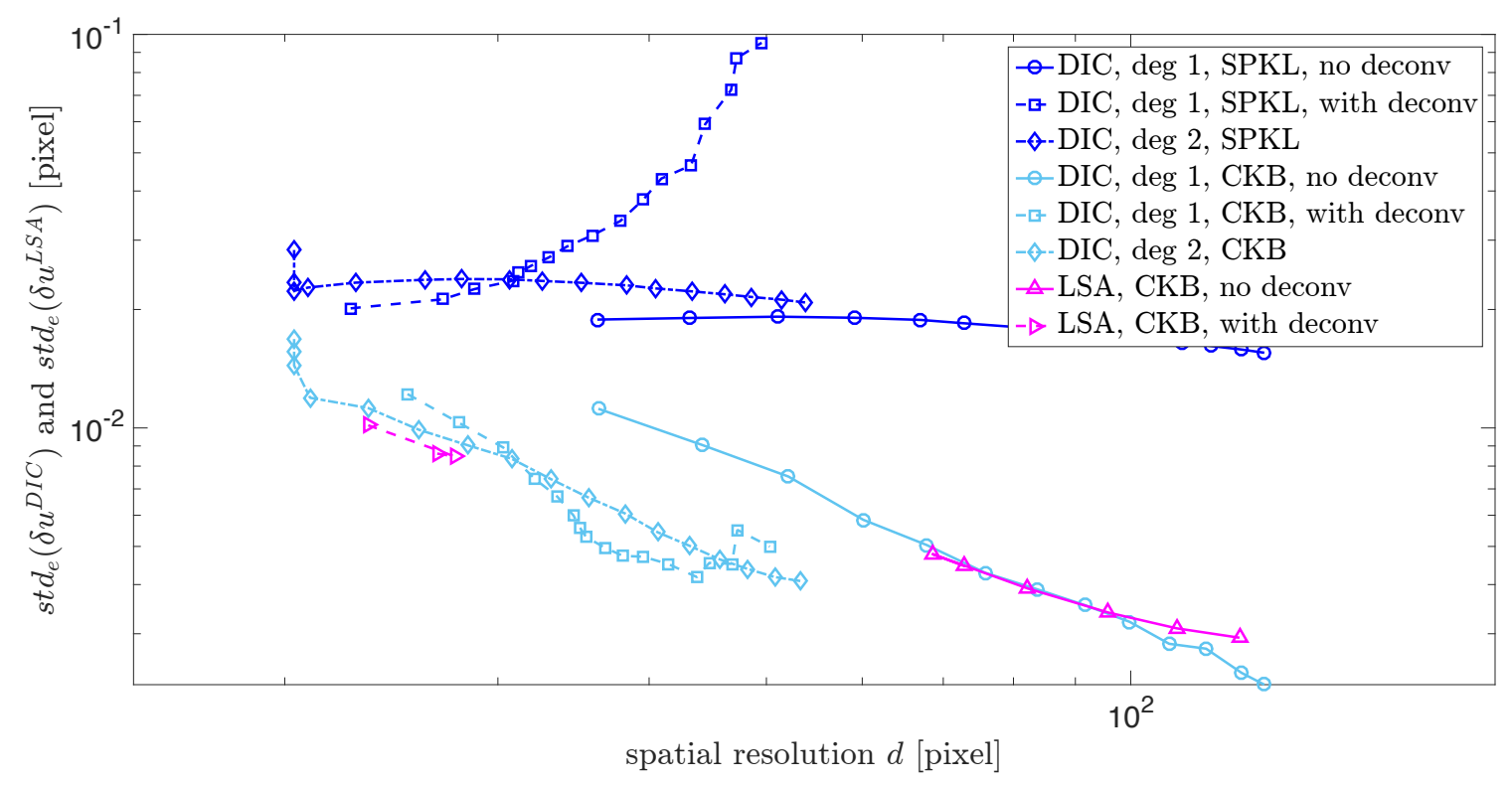

Figure 17: $\operatorname{std}_{e}\left(\delta u_{2}^{D I C}\right)$ and $\operatorname{std}_{e}\left(\delta u_{2}^{L S A}\right)$ as a function of the spatial resolution $d$ in the different cases studied in Figure 7.

\section{Computing time}

The objective here is to give a brief idea on the computing time needed for each technique to provide displacement fields. The computer used for these calculations is equipped with an Intel 8-core Intel Xeon E5-2620 v4 @ 2.1Ghz CPU and 64 Gb memory, and the number of iterations is set to 10 in the iterative deconvolution procedure for both DIC and LSA, which is conservative according to [33]. It is worth noting that the Matlab programs used in this program are not optimized, so the results provided here only give an idea of the order of magnitude of the computational cost in each case. We consider here all the cases discussed in Figure 11. Noiseless images are processed to estimate these computing times. The obtained results are shown in Figure 19. The number of points in the displacement maps obtained by DIC and LSA being not exactly the same, these computing times are normalized by dividing the total computing time obtained for each map by the number of measuring points in the corresponding displacement map. The most striking point is that the computing time for LSA is much lower than that observed for DIC (about one order of magnitude). The main reason is that DIC is iterative by essence, whereas LSA is quasidirect. It is quasi-direct and not direct because of the fixed-point algorithm used to retrieve the displacement field by subtracting the phases expressed in the same coordinate system in Equation 3. It is observed that only one iteration is sufficient in practice to converge, and 


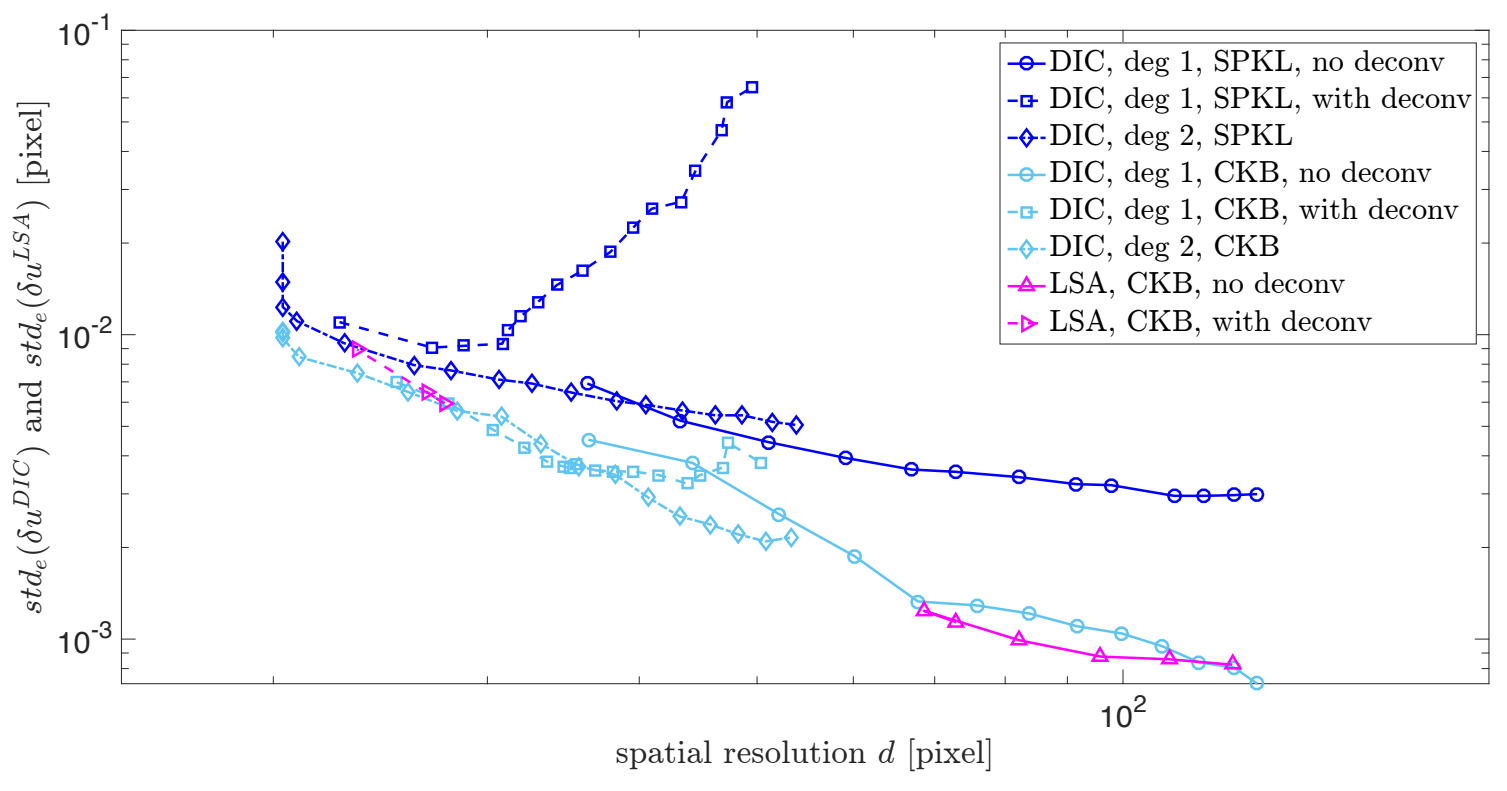

Figure 18: $s t d_{e}\left(\delta u_{2}^{D I C}\right)$ and $s t d_{e}\left(\delta u_{2}^{L S A}\right)$ as a function of the spatial resolution $d$ in the different cases studied in Figure 7 . Only the values of $\operatorname{std}_{e}\left(\delta u_{2}^{D I C}\right)$ and $\operatorname{std}_{e}\left(\delta u_{2}^{L S A}\right)$ at pixels corresponding of a displacement whose period is lower than the spatial resolution are considered in the calculation.

this iteration does not involve any heavy calculation. For DIC, interpolation is performed for each iteration at all the pixels of the subsets, which also notably increases the computing time. Another point is that the computing time increases while increasing $2 M+1$, thus while increasing the size of the subset in DIC, which is logical. Concerning LSA, we do not observe the same phenomenon when $\ell$ increases. This is due to the fact that the WFT used to extract the phase is a convolution, and this convolution becomes a mere multiplication in the Fourier domain. The Fourier transform of a Gaussian window is also a Gaussian window, but its size decreases as $\ell$ increases. However, this only marginally impacts the computing time. Finally, it is worth remembering that two maps are deconvolved with DIC for a pair of reference and currant images (the two in-plane displacement $u_{1}$ and $u_{2}$ ) but this number is equal to four with LSA (the two phases in both these images), see Section 3.3. It also means that if a whole series of images captured during a given test is deconvolved, the computing time needed to get each displacement map after the first of the series is divided by two compared to the values given in Figure 19-b, the two reference phases being deconvolved only once to get all the set of deconvolved displacement maps. 


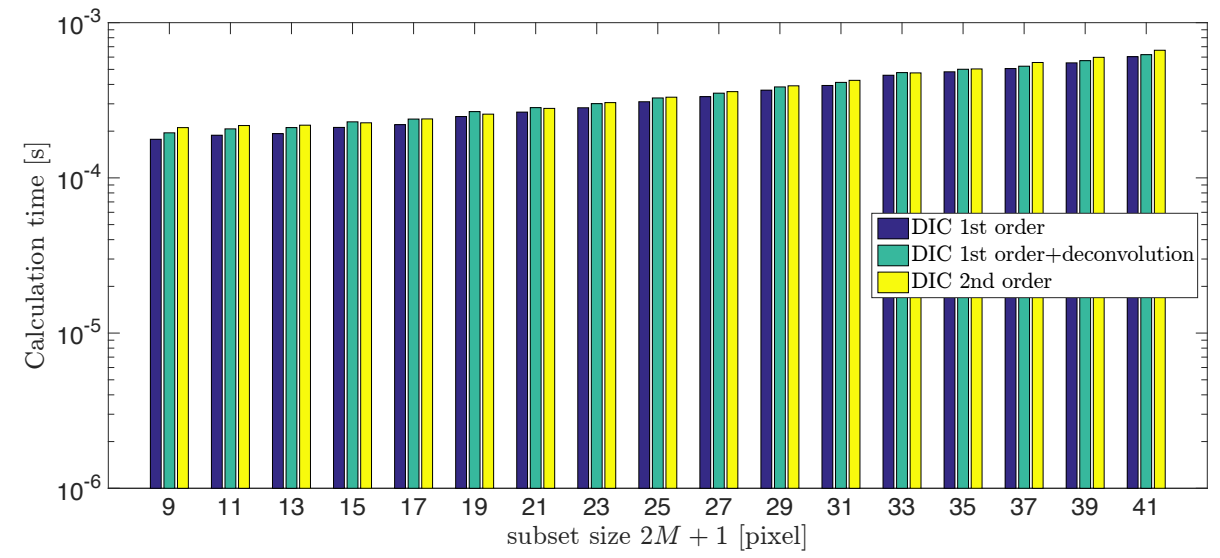

(a) DIC on speckle. DIC on checkerboard leads to similar computing times, so the corresponding results are not shown here

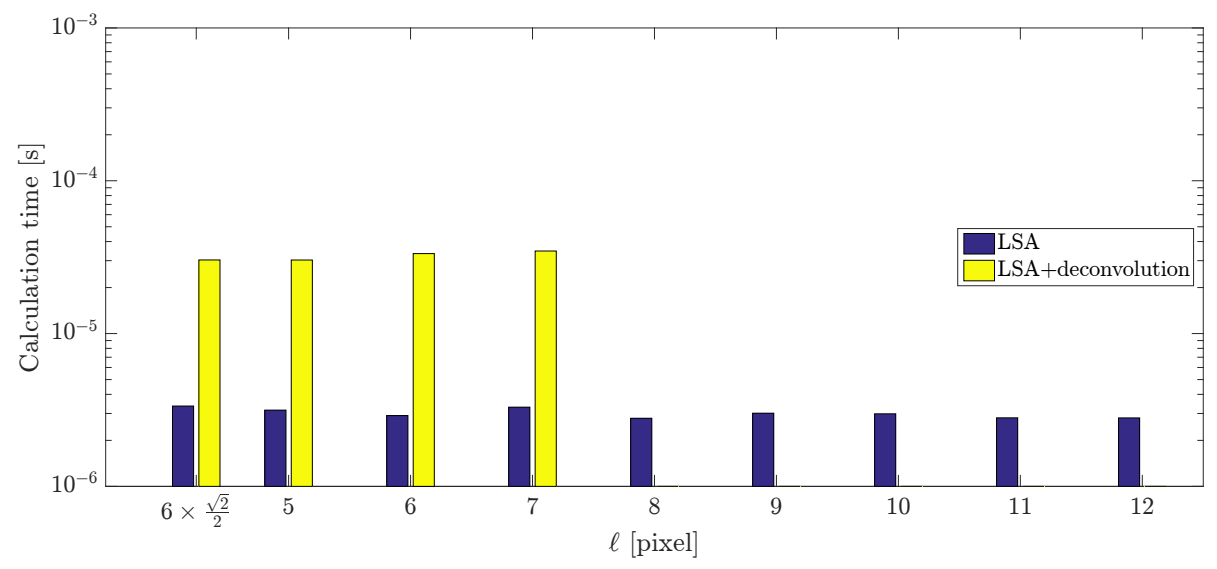

(b) LSA on checkerboard

Figure 19: computing time to obtain the noiseless maps used to plot the curves in Figure 11. 


\section{Experiment}

Before closing the paper, we briefly present the results given by an experiment in order to support some of the conclusions found above with synthetic images.

\subsection{Method}

We consider a translation test performed on two metallic square specimens (dimensions: $37 \times 31 \times 2 \mathrm{~mm}^{3}$ ). Each of them was marked with a different pattern. The first specimen was thoroughly spray-painted in white. Black droplets were then deposited to form the random pattern. A checkerboard of pitch $p=0.2 \mathrm{~mm}$ was transferred on the second specimen by using the technique proposed in [48]. The experimental setup used for this experiment is described in a recent paper [19], so the reader is referred to this reference for further details, in particular concerning the camera used for this experiment, the type of lighting, the device used to apply the translation and the support on which this device rests during the experiment. For the checkerboard, the magnification of the lens was adjusted in such a way that the pitch of the checkerboard was sampled over a number of pixels as close as possible to 6 pixels, which is the number of pixels used for the synthetic checkerboard above. The actual value for the pitch obtained in the experiment was 5.88 pixels, so this value is used when processing the images. The actual inclination of the checkerboard was $11.2 \mathrm{deg}$. The contrast in the images was optimized in such a way that the widest histogram of the gray level distribution was obtained in each case, and that the maximum value for the gray level was just under saturation. Compared to the simulations presented above, for which the contrast was set to 0.6 (thus meaning that $60 \%$ of the gray depth was used to encode the signal), the contrast is higher for the images of the real patterns. Figure 20 shows a closeup of each pattern.

With this experiment, the idea is to compare the noise level reached in the displacement map obtained with each technique. Deconvolution is not considered since no strain gradient occurs for a translation test. A set of 200 images was taken before and after applying the displacement. 200 displacement fields were then deduced for each type of pattern. The average displacement obtained for each of these displacement fields were then subtracted to get rid of potential micromovements than may corrupt the results, and the standard deviation of the displacement obtained at each pixel was deduced. The displacement resolution $\sigma_{u}$ was finally obtained by calculating the equivalent standard deviation over the whole field, which is defined by

$$
\sigma_{u}=\sqrt{\frac{1}{N} \sum_{k=1}^{N} s t d^{2}\left(u_{j}\left(x_{1}^{(k)}, x_{2}^{(k)}\right)\right)} \quad j=x_{1} \text { or } j=x_{2}
$$

where $N$ is the number of pixels in the experimental displacement maps, and $\operatorname{std}\left(u_{j}\left(x_{1}^{(k)}, x_{2}^{(k)}\right)\right), j=$ $x_{1}, x_{2}$ is the standard deviation of the distribution of the displacement $u_{j}$ at any pixel of 


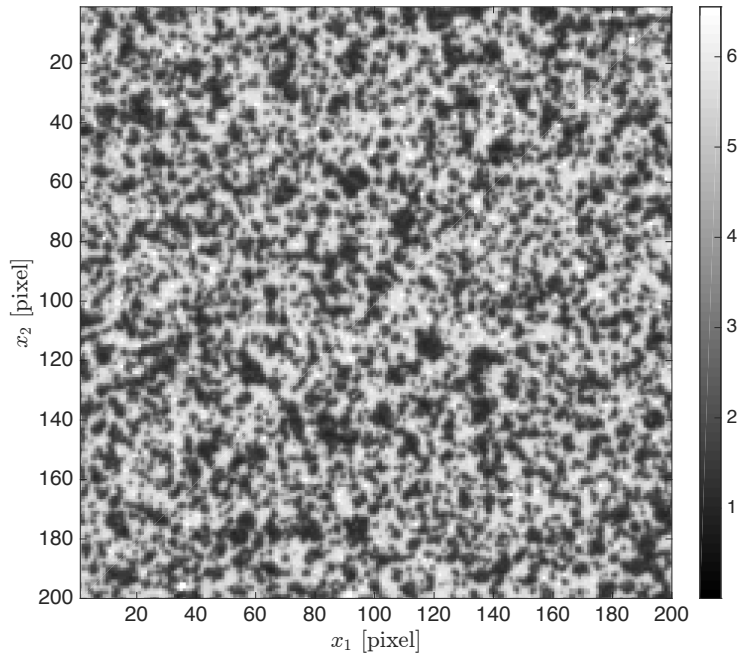

(a) Speckle

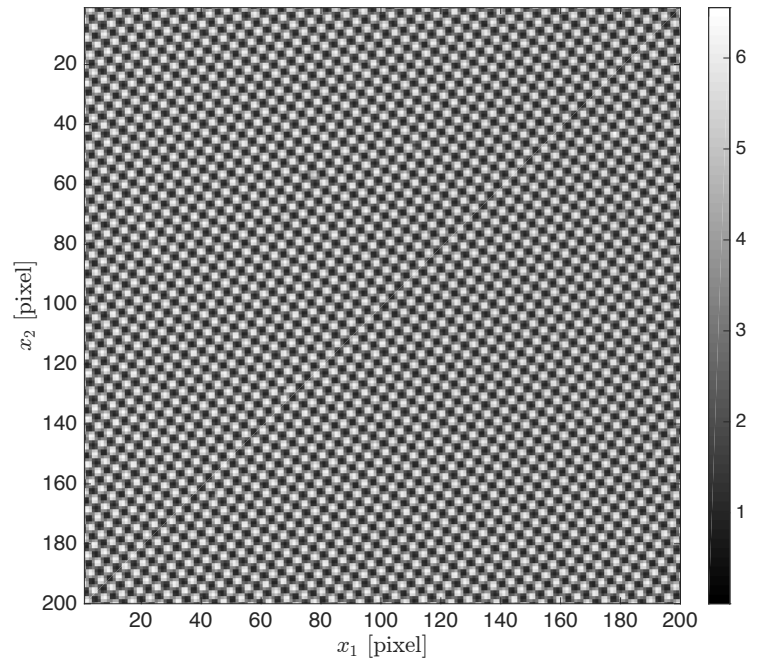

(b) Checkerboard

Figure 20: Closeup views of the real patterns used in this study.

coordinates $\left(x^{(k)}, y^{(k)}\right)$. The contrast in the images was the same for the two types of images, merely by adjusting the aperture of the lens and the shooting time of the camera. Typical results are presented here. The subset size $2 M+1$ is equal to 21 pixels and first-order matching functions are used. The standard deviation $\ell$ of the Gaussian window used to process the checkerboard images is thus set to $\ell=6.06$ pixels according to Equation 11, which gives the same bias $\lambda$ and the same spatial resolution $d$ for both techniques and patterns. The noise level in the displacement map can therefore be fairly compared between the two techniques. Note that changing the value of the subset size $2 M+1$ would automatically change the value of $\ell$ and thus the noise level in the displacement fields, but the ranking of the techniques would remain unchanged.

\subsection{Results}

The normalized histograms of $\operatorname{std}\left(u_{x_{1}}\right)$ and $\operatorname{std}\left(u_{x_{2}}\right)$ are displayed in Figure 21. For these experiment, $\sigma_{u}=5.1110^{-3}$ and $5.9010^{-3}$ along the $x_{1^{-}}$and $x_{2}$-directions, respectively, while $\sigma_{u}=7.5210^{-3}$ with the artificial speckle. $\sigma_{u}$ is greater for the simulated patterns because the contrast is lower in the artificial images than in the images taken during the experiment. Another remark is that the distributions are wider for the speckle pattern than for the checkerboard. Indeed, speckle patterns are by definition of random nature, which means that $\operatorname{std}\left(u_{1}\right)$ and $\operatorname{std}\left(u_{2}\right)$, more largely fluctuate, depending on the spatially changing value of the mean image gradient calculated over the subsets. 
(a1) Speckle and DIC, $2 M+1=21, \sigma_{u}=5.11 \mathrm{e}-03$

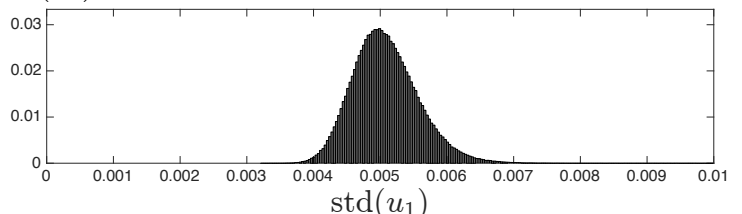

(b1) Speckle and DIC, $2 M+1=21, \sigma_{u}=5.90 \mathrm{e}-03$

(a2) Checkerboard and LSA, $\ell=6.06, \sigma_{u}=1.48 \mathrm{e}-03$
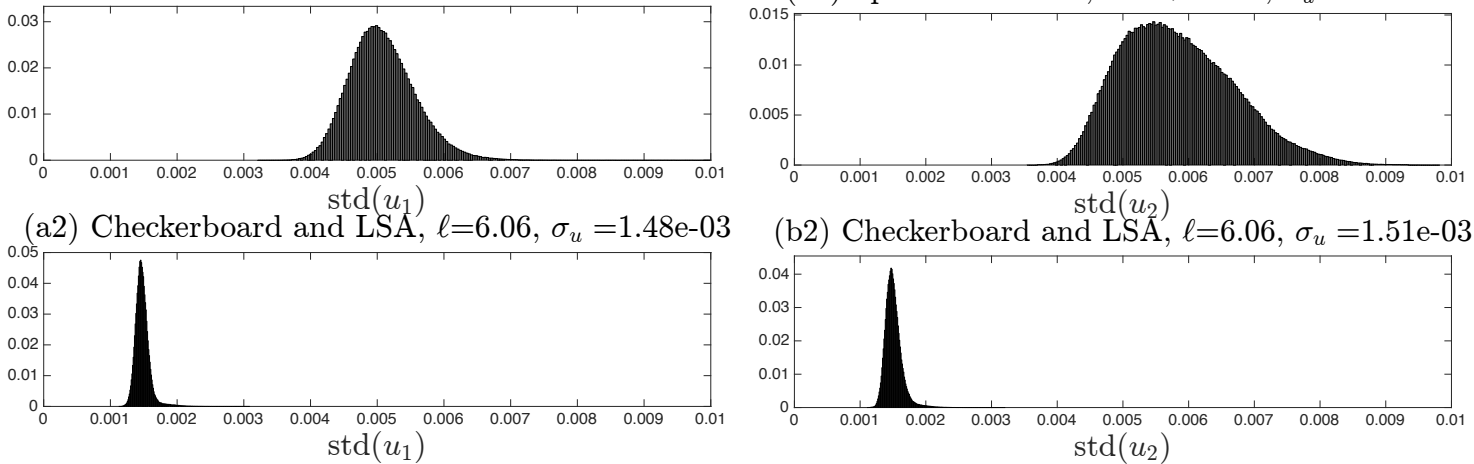

(b2) Checkerboard and LSA, $\ell=6.06, \sigma_{u}=1.51 \mathrm{e}-03$

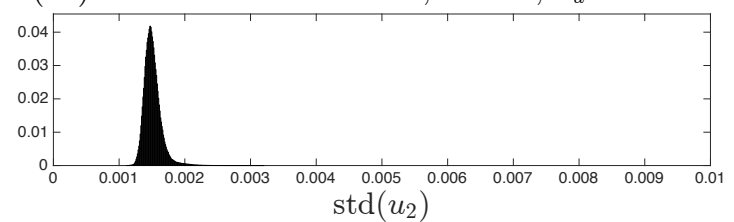

Figure 21: Normalized histogram of the noise in the displacement maps obtained with speckle and DIC (first line), and checkerboard and LSA (second line). The scale along the horizontal axis is the same for the four histograms.

The main point is that the ratio between $\sigma_{u}$ obtained with speckle and DIC on the one hand, and checkerboard and LSA on the other hand, is equal to 2.49 with the artificial images discussed in Section 4, while with the experimental results, this ratio is equal to 3.45 and 3.91 along the $x_{1}$ - and $x_{2}$-directions, respectively. The trend in the simulated and the experimental results is however the same since it is observed in both cases that $\sigma_{u}$ is much lower with checkerboards than with speckles. The difference in this ratio between results obtained with simulated and experimental patterns cannot be commented further, the random nature of the speckles automatically inducing fluctuating results with this type of pattern.

\section{Conclusion}

The effect of the pattern on the quality of the displacement field obtained by minimization of the optical residual was investigated in this paper. DIC was considered for the minimization of the optical residual over small regions in the spatial domain, and LSA for the minimization of the same quantity in the Fourier domain. Two different patterns were considered, namely a speckle typically used with DIC, and a checkerboard for LSA. The latter pattern leads to the highest possible image gradient if it is correctly sampled, so it is expected to lead to the lowest displacement resolution according to previous studies available in the literature. This was really observed in the numerical simulations performed in this paper. The residual obtained by subtracting the quantities returned by both techniques on the one hand, and the actual quantity convolved by kernel characterized for each technique in the literature on the other hand, was also considered in this comparison. This quantity is expected to reflect the influence of various effects which impair the results in 
addition to convolution which causes the amplitude of the frequencies involved in the true displacement and strain maps to be damped, with an intensity which increases with the frequency. The influence of the pattern, the interpolation error and the quantization of the signal are the most noticeable phenomena. Again, it was observed that the minimization of the optical residual in the Fourier domain on a checkerboard led to the best results. The price to pay to obtain displacement and strain fields by using checkerboard and LSA is the fact that depositing a regular pattern onto the specimen is more challenging than merely spraying a speckle.

\section{References}

[1] H. W. Schreier, M. Sutton, and A. Michael. Systematic errors in digital image correlation due to undermatched subset shape functions. Experimental Mechanics, 42(3):303$310,2002$.

[2] L. Yu and B. Pan. The errors in digital image correlation due to overmatched shape functions. Measurement Science and Technology, 26(4):045202, 2015.

[3] M. Bornert, F. Brémand, P. Doumalin, J.-C. Dupré, M. Fazzini, M. Grédiac, F. Hild, S. Mistou, J. Molimard, J.-J. Orteu, L. Robert, Y. Surrel, P. Vacher, and B. Wattrisse. Assessment of digital image correlation measurement errors: methodology and results. Experimental Mechanics, 49(3):353-370, 2009.

[4] W. Tong. Formulation of Lucas-Kanade digital image correlation algorithms for noncontact deformation measurements: a review. Strain, 49(4):313-334, 2013.

[5] Y. Su, Q. Zhang, Z. Gao, X. Xu, and X. Wu. Fourier-based interpolation bias prediction in digital image correlation. Optics Express, 23(15):19242-19260, 2015.

[6] J. Réthoré. A fully integrated noise robust strategy for the identification of constitutive laws from digital images. Internation Journal for Numerical Methods in Engineering, 84(6):631-660, 2010.

[7] B. Blaysat, M. Grédiac, and F. Sur. Effect of interpolation on noise propagation from images to DIC displacement maps. International Journal for Numerical Methods in Engineering, 108(3):213-232, 2016.

[8] G. Crammond, S.W. Boyd, and J.M. Dulieu-Barton. Speckle pattern quality assessment for digital image correlation. Optics and Lasers in Engineering, 51:1368-1378, 2013. 
[9] Y. Su, Q. Zhang, X. Xu, and Z. Gao. Quality assessment of speckle patterns for DIC by consideration of both systematic errors and random errors. Optics and Lasers in Engineering, 86:132-142, 2016.

[10] Y. L. Dong and B. Pan. A review of speckle pattern fabrication and assessment for digital image correlation. Experimental Mechanics, 57(8):1161-1181, 2017.

[11] Z. Chen, X. Shao, X. Xu, and X. He. Optimized digital speckle patterns for digital image correlation by consideration of both accuracy and efficiency. Applied Optics, $57(4): 884-893,2018$.

[12] G.F. Bomarito, J.D. Hochhalter, T.J. Ruggles, and A.H. Cannon. Increasing accuracy and precision of digital image correlation through pattern optimization. Optics and Lasers in Engineering, 91:73 - 85, 2017.

[13] B. Blaysat, M. Grédiac, and F. Sur. On the propagation of camera sensor noise to displacement maps obtained by DIC. Experimental Mechanics, 56(6):919-944, 2016.

[14] B. Pan, Z. Lu, and H. Xie. Mean intensity gradient: an effective global parameter for quality assessment of the speckle patterns used in digital image correlation. Optics and Lasers in Engineering, 48(4):469-77, 2010.

[15] D. Lecompte, A. Smits, S. Bossuyt, H. Sol, J. Vantomme, D. Van Hemelrijck, and A.M. Habraken. Quality assessment of speckle patterns for digital image correlation. Optics and Lasers in Engineering, 44(11):1132-1145, 2006.

[16] S. Bossuyt. Optimized patterns for digital image correlation. In Proceedings of the 2012 Annual Conference on Experimental and Applied Mechanics, Volume 3: Imaging Methods for Novel Materials and Challenging Applications, 2013.

[17] A. Lavatelli, R. Balcaen, E. Zappa, and D. Debruyne. Closed-loop optimization of DIC speckle patterns based on simulated experiments. IEEE Transactions on Instrumentation and Measurement, pages 1-11, 2019. On line (early access article). DOI: 10.1109/TIM.2019.2890890.

[18] M. Grédiac, B. Blaysat, and F. Sur. A critical comparison of some metrological parameters characterizing local digital image correlation and grid method. Experimental Mechanics, 57(3):871-903, 2017.

[19] M. Grédiac, B. Blaysat, and F. Sur. Extracting displacement and strain fields from checkerboard images with the localized spectrum analysis. Experimental Mechanics, 59(2):207-218, 2019.

[20] International vocabulary of metrology. Basic and general concepts and associated terms, 2008. Third edition. 
[21] A. Chrysochoos and Y. Surrel. Chapter 1. Basics of metrology and introduction to techniques. In M. Grdiac and F. Hild, editors, Full-field measurements and identification in solid mechanics, pages 1-29. Wiley, 2012.

[22] L. Wittevrongel, P. Lava, S. V. Lomov, and D. Debruyne. A self adaptive global digital image correlation algorithm. Experimental Mechanics, 55(2):361-378, 2015.

[23] DIC challenge: http://sem.org/dic-challenge/.

[24] J. Blaber, B. Adair, and A. Antoniou. Ncorr: Open-source 2d digital image correlation matlab software. Experimental Mechanics, 2015. doi: 10.1007/s11340-015-0009-1.

[25] C. Badulescu, M. Bornert, J.-C. Dupré, S. Equis, M. Grédiac, J. Molimard, P. Picart, R. Rotinat, and V. Valle. Demodulation of spatial carrier images: Performance analysis of several algorithms. Experimental Mechanics, 53(8):1357-1370, 2013.

[26] M. Grédiac, F. Sur, C. Badulescu, and J.-D. Mathias. Using deconvolution to improve the metrological performance of the grid method. Optics and Lasers in Engineering, 51(6):716-734, 2013.

[27] M. Sutton, J.J. Orteu, and H. Schreier. Image Correlation for Shape, Motion and Deformation Measurements. Basic Concepts, Theory and Applications. Springer, 2009.

[28] J. Neggers, B. Blaysat, J.P.M. Hoefnagels, and M.G.D. Geers. On image gradients in digital image correlation. International Journal for Numerical Methods in Engineering, 105(4):243-260, 2016.

[29] F. Sur and M. Grédiac. Influence of the analysis window on the metrological performance of the grid method. Journal of Mathematical Imaging and Vision, 56(3):472498, 2016.

[30] E. W. Grafarend. Linear and Nonlinear Models: Fixed Effects, Random Effects, and Mixed Models. Walter de Gruyter, 2006.

[31] M. Grédiac, F. Sur, and B. Blaysat. The grid method for in-plane displacement and strain measurement: a review and analysis. Strain, 52(3):205-243, 2016.

[32] F. Sur and M. Grédiac. Towards deconvolution to enhance the grid method for in-plane strain measurement. Inverse Problems and Imaging, 8(1):259-291, 2014.

[33] M. Grédiac, B. Blaysat, and F. Sur. A robust-to-noise deconvolution algorithm to enhance displacement and strain maps obtained by local DIC and LSA. Experimental Mechanics, 59(2):219-243, 2019. 
[34] B. Blaysat, J. Neggers, M. Grédiac, and F. Sur. Towards criteria characterizing the metrological performance of full-field measurement techniques. Application to the comparison between local and global versions of DIC. 2019. Submitted.

[35] P. Reu. All about speckles: Aliasing. Experimental Techniques, 38(5):1-3, 2014.

[36] F. Sur, B. Blaysat, and M. Grédiac. Rendering deformed speckle images with a Boolean model. Journal of Mathematical Imaging and Vision, 60(5):634-650, 2018.

[37] P. L. Reu, B. Blaysat, J. Helm, E. M.C. Jones, and M. Iadicola. Update on the DIC challenge 2.0 and the stereo-DIC challenge. In SEM annual conference "Expanding the Boundaries of Mechanics", Reno, USA, 2019. Proceedings of the conference.

[38] B. Pan, H. Xie, Z. Wang, K. Qian, and Z. Wang. Study on subset size selection in digital image correlation for speckle patterns. Optics Express, 16(10):7037-7048, 2008.

[39] Y. Q. Wang, M. Sutton, H. Bruck, and H. W. Schreier. Quantitative error assessment in pattern matching: effects of intensity pattern noise, interpolation, strain and image contrast on motion measurements. Strain, 45(2):160 -178, 2009.

[40] F. Sur, B. Blaysat, and M. Grédiac. Determining displacement and strain maps immune from aliasing effect with the grid method. Optics and Lasers in Engineering, 86:317-328, 2016.

[41] A. Foi, M. Trimeche, V. Katkovnik, and K. Egiazarian. Practical Poissonian-Gaussian noise modeling and fitting for single-image raw-data. IEEE Transactions on Image Processing, 17(10):1737-1754, 2008.

[42] F. Hild and S. Roux. Comparison of local and global approaches to digital image correlation. Experimental Mechanics, 52(9):1503-1519, 2012.

[43] A. Savitzky and M.J.E. Golay. Smoothing and differentiation of data by simplified least-squares procedures. Analytical Chemistry, 36(3):1627-1639, 1964.

[44] R.B. Lehoucq, P.L. Reu, and D.Z. Turner. The effect of the ill-posed problem on quantitative error assessment in digital image correlation. Experimental Mechanics, 2017. Accepted.

[45] S. Fayad and P. L. Reu. Pattern-induced bias in out-of-plane motion in digital image correlation. In SEM annual conference "Expanding the Boundaries of Mechanics", Reno, USA, 2019. Proceedings of the conference.

[46] S.S. Fayad, D.T. Seidl, and P.L. Reu. Spatial DIC errors due to pattern-induced bias and grey level discretization. Experimental Mechanics, 2019. Accepted, doi: 10.1007/s11340-019-00553-9. 
[47] F. Sur, B. Blaysat, and M. Grédiac. On biases in displacement estimation for image registration, with a focus on photomechanics. 2019. Submitted.

[48] J.L. Piro and M. Grédiac. Producing and transferring low-spatial-frequency grids for measuring displacement fields with moiré and grid methods. Experimental Techniques, 28(4):23-26, 2004.

\section{Appendix}

The aim of this section is to show how to determine the value of $\ell$ used in LSA, which is equivalent to the value of $2 M+1$ used in DIC. "Equivalent" means here that DIC used with a subset size equal to $2 M+1$ features the same bias and spatial resolution as LSA used with a standard deviation equal to $\ell$. As mentioned earlier, it does not mean that the outputs of DIC and LSA with these equivalent parameters give the same estimates of a real displacement field featuring strain gradients.

Predicting the bias for given values of $2 M+1$ and $\ell$ can be made as follows. Assuming the real (and sought) displacement is a sine function of amplitude $A$, the bias as defined in Section 2 can be directly estimated with the transfer function of the filter characterizing the measuring technique. This filter is the Savitzky-Golay filter for DIC [1] and a Gaussian filter for LSA [18]. By definition, the transfer function of the filter is equal to its Fourier transform. Indeed, in linear translation-invariant signal processing, the transfer function of any linear filter is defined as the Fourier transform of its impulse response.

\section{Case of DIC}

With DIC, we deal by definition with the discrete form of the transfer function, which can be written as follows as a function of the frequency $f$ of the prescribed displacement

$$
\widehat{h}^{D I C}(f)=h(0)+2 \sum_{i=1}^{M} h(i) \cos (2 i \pi f)
$$

Since we deal with images, we have sampled signals. Hence $f$ lies in this equation between 0 and the Nyquist frequency, equal here to 0.5 pixel $^{-1}$. The $h(i)$ coefficients, $i \in\left[\begin{array}{ll}0 & M\end{array}\right]$, are the Savitzky-Golay coefficients defined in [43] for DIC, as explained in [1] and demonstrated in [47]. These coefficients depend on $M$ and on the degree of the matching function used to express the displacement field within the subset. In the common case, for which the matching function is linear, the Savitzky-Golay coefficients are all equal to $\frac{1}{2 M+1}[43]$. 


\section{Case of LSA}

With LSA, the same discrete form could be chosen for the Fourier transform, but a continuous form of the Fourier transform is also available, which is not the case for DIC. This continuous form can therefore be used for the sake of convenience. Since a Gaussian window of standard deviation $\ell$ is used here in the WFT, the Fourier transform of the filter used with LSA is also a Gaussian function. Indeed, the Fourier transform of a Gaussian function is also a Gaussian function, which is a classic result in signal processing. This transfer function can be written as follows [31]:

$$
\widehat{h}^{L S A}(f)=e^{-2 \pi^{2} \ell^{2} f^{2}}
$$

\section{Equivalence between $2 M+1$ and $\ell$ for a given value of $\lambda$}

As a consequence of the two preceding equations, finding the standard deviation of a Gaussian window used in LSA, which is equivalent, in terms of bias and spatial resolution, to a subset size $2 M+1$ used in DIC, consists of the following steps:

1. choosing the same value for the bias $\lambda$ for both DIC and LSA, which gives:

$$
\widehat{h}^{D I C}(f)=\widehat{h}^{L S A}(f)=1-\lambda
$$

2. for a given subset size $2 M+1$, finding numerically (no analytical solution is available) the highest frequency $f$, which is the solution of Equation 13. Thus

$$
h(0)+2 \sum_{i=1}^{M} h(i) \cos (2 i \pi f)=1-\lambda
$$

3. inverting Equation 14 in order to deduce the corresponding standard deviation $\ell$ for the Gaussian window used in the WFT. Since $d=\frac{1}{f}$, we have

$$
\ell=\frac{d}{\pi} \sqrt{\frac{-\log (1-\lambda)}{2}}, \quad \text { with } 0<\lambda<1
$$

\section{Influence of $\lambda$}

Choosing a bias $\lambda=10 \%$ for both techniques is somewhat arbitrary. Changing the value of the bias, for instance by considering $\lambda=5 \%$ and $\lambda=20 \%$ instead of $\lambda=10 \%$, has a great impact on the spatial resolution $d$ but does not really influence the standard deviation $\ell$ of the Gaussian window used in the WFT, as illustrated in the examples shown in Table 3 in a particular (but representative) case, namely $2 M+1=21$ pixels. $d$ and $\ell$ are calculated for 
various values of $\lambda$ fixed a priori. It can be seen that the value of $\ell$ equivalent to $2 M+1$ is nearly unaffected when the bias $\lambda$ changes. This makes the results nearly independent of this arbitrary choice.

\begin{tabular}{|c|c|c|}
\hline $\begin{array}{c}\text { bias } \lambda \\
\text { for DIC and LSA }\end{array}$ & $\begin{array}{c}\text { spatial resolution } d \\
\text { for DIC and LSA } \\
{[-]}\end{array}$ & $\begin{array}{c}\ell \text { used in LSA } \\
\text { corresponding to } 2 M+1=21 \text { pixels } \\
\text { for the subset size used in DIC } \\
\text { [pixel] }\end{array}$ \\
\hline $5 \%$ & 165.08 & 6.09 \\
$10 \%$ & 83.74 & 6.12 \\
$20 \%$ & 58.24 & 6.19 \\
\hline
\end{tabular}

Table 3: Values of $\ell$ used in LSA leading to the same spatial resolution and the same bias as those obtained in DIC with a subset size equal to $2 M+1=21$ pixels.

\section{Particular case of small values of $\lambda$ and DIC used with linear matching functions}

We demonstrate here that if linear matching functions are considered in DIC, a closedform expression giving $\ell$ as a function of $M$ is available. In addition, $\ell$ does not depend, at first approximation, on the bias $\lambda$ when $\lambda$ is small. We consider first again Equation 16 . Assuming that $2 M \pi f$ is small enough, the cosines involved in this equation can be replaced by their third-order approximation. Thus Equation 16 becomes

$$
h(0)+2 \sum_{i=1}^{M} h(i)\left(1-\frac{(2 i \pi f)^{2}}{2}\right)=1-\lambda
$$

Since $h(0)+2 \sum_{i=1}^{M} h(i)=1[43]$, we have

$$
4 \pi^{2} f^{2} \sum_{i=1}^{M} i^{2} h(i)=\lambda
$$

Let us now focus on linear matching functions. In this case the Savitzky-Golay coefficients are all equal to $h(i)=\frac{1}{2 M+1}, \forall i \in\{1 \ldots M\}$ [43]. Hence

$$
\sum_{i=1}^{M} i^{2} h(i)=\frac{1}{2 M+1} \sum_{i=1}^{M} i^{2}
$$


According to the Faulhaber's formula, we have $\sum_{i=1}^{M} i^{2}=\frac{2 M^{3}+3 M^{2}+M}{6}$, so after simplification, we can deduce from Equation 19

$$
f=\frac{1}{2 \pi} \sqrt{\frac{6 \lambda}{M(M+1)}}
$$

It can be checked that the third-order approximation of the cosines made in Equation 18 still remains acceptable for significant values of $M$. For instance, with $M=10$, which is the case considered in the examples discussed in this paper, the error made by substituting the quantity corresponding to the greatest value of the index $i$ of the sum is equal to $11 \%$. The error made on the other terms of this approximated sum is automatically lower. In addition, the weight of these terms in the sum is higher than the weight of the last term, the cosine being a decreasing function just after 0 .

Plugging Equation 21 into Equation 17, where $f=\frac{1}{d}$, leads to

$$
\ell=\sqrt{\frac{M(M+1)}{3}} \times \sqrt{\frac{-\log (1-\lambda)}{\lambda}}, \quad \text { with } 0<\lambda<1
$$

If $\lambda$ is small, then $\frac{-\log (1-\lambda)}{\lambda} \simeq 1$ and Equation 22 reduces to

$$
\ell=\sqrt{\frac{M(M+1)}{3}}
$$

For instance, in the case $2 M+1$ considered in Table 3 above, Equation 23 gives $\ell=$ 6.05 pixels, which is very close to the values given in this table for various values of $\lambda$. This result shows that in the particular case of DIC used with linear matching functions, $\ell$ does not really depend on $\lambda$ when $\lambda$ is small. $\ell$ and thus the width of the Gaussian window used in LSA only depend on the size of the subset $2 M+1$ used in DIC. 